\title{
BULGELESS GALAXIES AT INTERMEDIATE REDSHIFT: SAMPLE SELECTION, COLOR PROPERTIES, AND THE EXISTENCE OF POWERFUL ACTIVE GALACTIC NUCLEI
}

\author{
Luca Bizzocchi ${ }^{1}$, Mercedes E. Filho $^{2}$, Elvira Leonardo ${ }^{1}$, Marco Grossi $^{1,3}$, Roger L. Griffith $^{4}$, \\ José Afonso $^{1,3}$, Cristina Fernandes ${ }^{1}$, João Retrê ${ }^{1}$, Sonia Anton ${ }^{5,6}$, Eric F. Bell ${ }^{7}$, \\ Jarle Brinchmann $^{8}$, Bruno Henriques ${ }^{9}$, Catarina Lobo ${ }^{2,10}$, and Hugo Messias ${ }^{11}$ \\ ${ }^{1}$ Centro de Astronomia e Astrofísica da Universidade de Lisboa, Observatório Astronómico de Lisboa, Tapada da Ajuda, 1349-018 Lisbon, Portugal \\ ${ }^{2}$ Centro de Astrofísica da Universidade do Porto, Rua das Estrelas, 4150-762 Porto, Portugal \\ ${ }^{3}$ Department of Physics, Faculty of Sciences, University of Lisbon, Campo Grande, 1749-016 Lisbon, Portugal \\ ${ }^{4}$ Infrared Processing and Analysis Center, California Institute of Technology, Pasadena, CA 91125, USA \\ ${ }^{5}$ Centro de Investigação em Ciências Geo-Espaciais, Faculdade de Ciências da Universidade do Porto, Porto, Portugal \\ ${ }^{6}$ SIM, Faculdade de Ciências da Universidade de Lisboa, Lisboa, Portugal \\ ${ }^{7}$ Department of Astronomy, University of Michigan, 500 Church Street, Ann Arbor, MI 48109, USA \\ ${ }^{8}$ Leiden Observatory, Leiden University, P.O. Box 9513, NL-2300 RA Leiden, The Netherlands \\ ${ }^{9}$ Max-Planck-Institut für Astrophysik, Karl Schwarzschild Straße 1, D-85748 Garching bei München, Germany \\ ${ }^{10}$ Departamento de Física e Astronomia, Faculdade de Cências da Universidade do Porto, Rua do Campo Alegre 687, 4169-007 Porto, Portugal \\ ${ }^{11}$ Departamento de astronomía, Av. Esteban Iturra 6to piso, Facultad de Ciencias Físicas y Matemáticas, Universidad de Concepción, Chile \\ Received 2013 July 31; accepted 2013 December 14; published 2014 January 22
}

\begin{abstract}
We present a catalog of bulgeless galaxies, which includes 19,225 objects selected in four of the deepest, largest multi-wavelength data sets available_COSMOS, AEGIS, GEMS, and GOODS—at intermediate redshift $(0.4 \leqslant z \leqslant 1.0)$. The morphological classification was provided by the Advanced Camera for Surveys General Catalog (ACS-GC), which used publicly available data obtained with the ACS instrument on the Hubble Space Telescope. Rest-frame photometric quantities were derived using kcorrect. We analyze the properties of the sample and the evolution of pure-disk systems with redshift. Very massive $\left[\log \left(M_{\star} / M_{\odot}\right)>10.5\right]$ bulgeless galaxies contribute to $\sim 30 \%$ of the total galaxy population number density at $z \geqslant 0.7$, but their number density drops substantially with decreasing redshift. We show that only a negligible fraction of pure disks appear to be quiescent systems, and red sequence bulgeless galaxies show indications of dust-obscured star formation. X-ray catalogs were used to search for X-ray emission within our sample. After visual inspection and detailed parametric morphological fitting we identify 30 active galactic nuclei (AGNs) that reside in galaxies without a classical bulge. The finding of such peculiar objects at intermediate redshift shows that while AGN growth in merger-free systems is a rare event $(0.2 \%$ AGN hosts in this sample of bulgeless galaxies), it can indeed happen relatively early in the history of the universe.
\end{abstract}

Key words: catalogs - galaxies: evolution - galaxies: structure - X-rays: galaxies

Online-only material: color figures

\section{INTRODUCTION}

One of the cornerstones of modern cosmology is understanding the formation and evolution of galaxies and the relative importance of merger processes. According to Cold Dark Matter $(\Lambda \mathrm{CDM})$ structure formation models, galaxies form hierarchically: dark matter collapses into halos in the early universe, which virialize and cluster hierarchically to form large-scale structures. Rotating disks start forming in slowly growing dark matter halos (Fall \& Efstathiou 1980) and become the building blocks of galaxies (Cole et al. 2000). In this framework of a merger-dominated universe, galaxies without a bulge are difficult to produce: the disks will thicken or warp due to interactions between low mass halos (Chambers et al. 2004) or be completely disrupted by major mergers (Koda et al. 2009), leaving behind a classical bulge. Halos that somehow escape or at least avoid major mergers since $z \sim 3$ could, in principle, form bulgeless galaxies, especially if some form of heuristic prescription for stellar feedback is included in the simulations (D'Onghia \& Burkert 2004; Robertson et al. 2004), but the simulated disks end up being smaller, denser, and having lower angular momentum than the observed ones. However, more recent hydrodynamical simulations of dwarf galaxies show that outflows can prevent the formation of classical merger-driven bulges in low mass systems by expelling most of the low angular momentum gas generated in such events (Brook et al. 2012; Dutton \& van den Bosch 2009).

The observed correlations between black hole $(\mathrm{BH})$ mass and host galaxy properties (such as stellar velocity dispersion, circum-nuclear star formation, mass, and luminosity; see, e.g., Kormendy \& Richstone 1995; Magorrian et al. 1998; Tremaine et al. 2002; Häring \& Rix 2004; Koda et al. 2009; Schawinski et al. 2010) are rarely observed in galaxies with no bulges (Kormendy et al. 2011, hereafter K11), thus suggesting that the formation and evolution of galaxy bulges are linked to the formation and evolution of the host BHs, albeit perhaps differently in early- and late-type objects (Schawinski et al. 2010).

The growth of a BH in a bulgeless galaxy remains difficult to understand in the absence of merger processes. On the other hand, several observations of BHs and active galactic nuclei (AGNs) in nearby bulgeless galaxies currently indicate that a classical bulge is not a requirement for an (active) nuclear BH: NGC 4395 (Filippenko \& Ho 2003; Peterson et al. 2005) is a bulgeless $\mathrm{Sm}$ galaxy with a Seyfert 1 spectrum, Pox 52 (Thornton et al. 2008) is a dwarf Seyfert 1 galaxy, Henize 2-10 
is the first ever (irregular) dwarf discovered to have a supermassive BH (SMBH; Reines et al. 2011), whereas NGC 3621, NGC 4178, NGC 3367, NGC 4536, and NGC 4561 (Satyapal et al. 2007; Barth et al. 2009; Satyapal et al. 2009; Secrest et al. 2012; McAlpine et al. 2011; Araya Salvo et al. 2012), are latetype Sd galaxies with AGN activity from low-mass BHs. Also, Desroches \& Ho (2009) found a high incidence of AGN activity in a sample of late-type spirals observed with Chandra, indicating that BHs can evolve in galaxies with little or no evidence for bulges.

Kormendy et al. (K11) found no correlation between $\mathrm{BH}$ and disk masses in a sample of 11 galaxies. Mathur et al. (2012) analyzed ten pseudo-bulge Narrow Line Seyfert 1 (NLS 1) galaxies and compared the $\mathrm{BH}$ mass to the pseudo-bulge luminosity, finding that the BHs were undermassive compared to the host galaxies. This supports the hypothesis that BHs in pseudo-bulges/disk galaxies are in the growth mode at the present epoch and that this growth is triggered by secular processes. In this context, BHs/AGNs found in bulgeless galaxies are thought to be the smallest BHs grown by local processes and are the seeds of the larger merger-grown SMBHs found in massive galaxies (K11).

The Sloan Digital Sky Survey (SDSS) has been used to establish a benchmark for the local universe (Kautsch et al. 2006) and to investigate the significance of AGN feedback in bulgeless galaxies, including quenching of star formation (Bell 2008; Coelho et al. 2013). Recently, a sample of 13 AGNs in bulgeless galaxies with $\mathrm{BH}$ masses in the range $10^{6}-10^{7} M_{\odot}$ was selected from the SDSS using visual classification from the Galaxy Zoo (Simmons et al. 2012). The authors showed that significant $\mathrm{BH}$ growth can take place in the absence of mergers or violent disk instabilities.

Given the importance of bulgeless galaxies to understand galaxy evolution, a study of these objects at higher redshifts is fundamental. The existence of a set of very deep and highresolution observations with the Hubble Space Telescope (HST) allows to extend morphological local studies, as those performed with SDSS, to much higher redshifts, and start mapping the evolution of bulgeless galaxies. Here we present a cata$\log$ and a first analysis of such systems at intermediate redshift $(0.4 \leqslant z \leqslant 1.0)$, using publicly available data from four of the largest, deepest multi-wavelength surveys: the Cosmological Evolutionary Survey ${ }^{12}$ (COSMOS; Scoville et al. 2007), the All-wavelength Extended Groth Strip (EGS) International Survey $^{13}$ (AEGIS; Davis et al. 2007), the Galaxy Evolution from Morphology and SEDs (GEMS) survey ${ }^{14}$ (Caldwell et al. 2008), and the Great Observatories Origins Deep Survey ${ }^{15}$ (GOODS; Dickinson et al. 2003; Giavalisco et al. 2004). The latter survey targets two separate fields, the Hubble Deep Field North (HDF-N, hereafter referred to as GOODS-N) and the Chandra Deep Field South (CDF-S, hereafter referred to as GOODS-S). The HST data for all these fields have been recently assembled by Griffith et al. (2012) into a single homogeneously analyzed data set.

Besides high-resolution optical imaging, these fields also possess very comprehensive multi-wavelength coverage, from $\mathrm{X}$-rays to radio, as well as vast spectroscopic information. The synergy of such a panchromatic data set offers us the opportunity to investigate the significance of bulgeless galaxies

\footnotetext{
12 http://cosmos.astro.caltech.edu

13 http://aegis.ucolick.org

14 http://www.mpia.de/GEMS/gems.htm

15 http://www.stsci.edu/science/goods/
}

Table 1

ACS Galaxy Catalog Survey Fields

\begin{tabular}{lccccc}
\hline \hline Survey & $\begin{array}{c}\text { R.A. } \\
\text { J2000 }\end{array}$ & $\begin{array}{c}\text { Decl. } \\
\text { J2000 }\end{array}$ & $\begin{array}{c}\text { Area } \\
\left(\mathrm{deg}^{2}\right)\end{array}$ & Filters & $\begin{array}{c}\text { Pixel Scale } \\
\left(\operatorname{arcsec~} \text { pixel }^{-1}\right)\end{array}$ \\
\hline COSMOS & $10: 00: 28$ & $+02: 12: 21$ & 1.8 & F814W & 0.05 \\
AEGIS & $14: 17: 00$ & $+52: 30: 00$ & 0.197 & F606W \& F814W & 0.03 \\
GEMS & $03: 32: 25$ & $-27: 48: 50$ & 0.21 & F606W \& F850LP & 0.03 \\
GOODS-S & $03: 32: 30$ & $-27: 48: 20$ & 0.07 & F606W \& F850LP & 0.03 \\
GOODS-N & $12: 36: 55$ & $+62: 14: 15$ & 0.07 & F606W \& F775W & 0.03 \\
\hline
\end{tabular}

and bulgeless AGN hosts when the universe was less than one-half of its current age (more than 7 Gyr in the past), an epoch much more active than the current one. This can provide valuable insight into the understanding of the evolutionary path of bulgeless systems within the overall galaxy formation and evolutionary scenario.

The paper is organized as follows: in Sections 2 and 3 we describe the data set and present the derivation of the relevant rest-frame quantities; in Section 4 we present the final magnitude-limited $\left(m_{\mathrm{AB}}<24\right)$ catalog containing 19,225 bulgeless galaxies at intermediate redshift $(0.4 \leqslant z \leqslant 1.0)$, and investigate the reliability of the adopted morphological classification, as well as the accuracy of the mass estimates. In Section 5 an overview of the sample properties, focusing on bulgeless galaxy evolution and on peculiar subsamples, such as red and massive bulgeless galaxies, is provided. In Section 6 we present the first systematic search for bulgeless AGN at intermediate redshift, based on X-ray emission, and presenting an accurate analysis of these objects' morphology. Finally, a summary of this work is given in Section 7.

Throughout the article we use $H_{0}=71 \mathrm{~km} \mathrm{~s}^{-1} \mathrm{Mpc}^{-1}$, $\Omega_{\mathrm{M}}=0.27$, and $\Omega_{\Lambda}=0.73$ (WMAP7; Larson et al. 2011). All magnitudes are (unless otherwise noted) expressed in the AB system.

\section{SAMPLE SELECTION}

The present study is based on the Advanced Camera for Surveys (ACS) HST public morphology catalog, which has been recently assembled by Griffith et al. (2012), merging the largest HST imaging surveys. The catalog contains a total of 469,501 sources in five sky fields: COSMOS (304,688 sources), AEGIS (70,142 sources), GEMS (59,447 sources), GOODS-S (10,999 sources), and GOODS-N (24,225 sources). This large ACS data set has been homogeneously analyzed using the code GALAPAGOS (Barden et al. 2012), an application that combines object detection with SEXTRACTOR (Bertin \& Arnouts 1996) and light profile modeling with GALFIT (Peng et al. 2002). These data provide the basis to extract precise morphological parameters for the galaxies. A thorough description of the ACS General Catalog (henceforth referred to as the ACS-GC), its contents, additional data products, and details of its construction from $H S T$ /ACS images, are given in Griffith et al. (2012). A brief summary of the ACS-GC imaging data is also reported here in Table 1, giving central coordinates for the surveys, survey size, filters, and pixel scales. In the following, we outline those details which are pertinent to the context of the present investigation.

\subsection{Imaging and Morphology Data}

The morphological classification was derived from the analysis of the surface brightness profiles, obtained at the reddest filter image available for each field, namely $I_{\mathrm{F} 814 \mathrm{~W}}$ for the 
COSMOS and EGS fields, $z_{\mathrm{F} 850 \mathrm{LP}}$ for the GEMS + GOODS-S fields, and $i_{\mathrm{F} 775 \mathrm{~W}}$ for the GOODS-N field. The light distribution of the galaxies was analyzed by fitting a single Sérsic profile (Sérsic 1968):

$$
\Sigma(r) \propto \exp \left\{-\kappa\left(r / r_{e}\right)^{-1 / n}-1\right\}
$$

where the Sérsic index $n$ describes the shape of the light profile, $r_{e}$ is the effective radius of the galaxy, and $\kappa$ is a positive parameter that is coupled to $n$, such that half of the total flux is always within $r_{e}$. An index $n=1$ corresponds to an exponential profile of a typical pure-disk galaxy, whereas $n=4$ corresponds to the de Vancouleurs' profile of elliptical or spheroidal galaxies.

In order to improve the robustness of the morphology selection, we rejected the faintest galaxies by imposing a magnitude cut, $m_{\mathrm{AB}} \leqslant 24$, in the filter considered for each field (see above). To avoid sources with very little or no morphological information, we removed point sources from the sample in the following way. Pixel sizes in the adopted surveys range from 0.05 arcsec for COSMOS (ACS/Wide Field Channel), to 0.03 arcsec (ACS/High Resolution Channel) for AEGIS, GEMS, and GOODS. For an average ACS point-spread function (PSF) of 0.075 arcsec, point-like sources occupy just over one to two pixels. We thus discarded catalog entries with a half-light radius smaller than 0.15 arcsec, corresponding to two times the PSF. We further discarded all sources with a $b / a$ axis ratio less than 0.5 (equivalent to inclinations larger than $60 \mathrm{deg}$ ), in order to minimize the effects of dust extinction on galaxy colors. At this stage the galaxy sample contains 78,830 sources.

It should be considered that galaxy appearance may depend on the rest-frame wavelength at which it is observed, and thus comparison of the morphology of galaxies observed at different redshift or through different filters should be treated with additional care. Given the redshift interval under study $(0.4 \leqslant z \leqslant 1.0)$ and the filters adopted, we are sampling a rest-frame range of $\lambda 3900-6100 \AA$, where the morphological $K$-correction is found to be not significant for most galaxies. This has been pointed out in the detailed COSMOS morphological study of Scarlata et al. (2007; see also, detailed discussions in Lotz et al. 2004; Cassata et al. 2005).

\subsection{Redshift Data}

The ACS-GC contains spectroscopic and photometric redshifts from different sources, as summarized in the following (see Griffith et al. 2012, for details).

1. COSMOS: $\sim 10,300$ spectroscopic redshifts are provided from the VIMOS/VLT zCOSMOS survey ${ }^{16}$ (Lilly et al. 2007). This data release provides a classification for the quality of the spectroscopic measurements: in the present work we considered classes 3 and 4 (very secure redshifts, $P \geqslant 99.5 \%$ ), class 2 (probable redshift, $P>92 \%$ ), and class 9 (one identified line, high photo- $z$ consistency) as reliable, whereas other redshift classes were discarded. Photometric redshifts $(\sim 252,000)$ are from the COSMOS public catalog (Ilbert et al. 2009), and are based on 30 band photometry, which spans the wavelength range from the ultraviolet to the mid-infrared.

2. AEGIS: the ACS catalog provides 5700 spectroscopic redshifts from the Deep Extragalactic Evolutionary probe,

16 http://archive.eso.org/cms/eso-data/data-packages/zcosmos-datarelease-dr2/
DEEP $2^{17}$, obtained with DEIMOS on Keck (Davis et al. 2003, 2007). Following the DEEP2 team's recommendations, only the entries with redshift quality $\left(z_{q}\right)$ greater than or equal to $3(P>90 \%)$ were treated as having a reliable redshift measurement. Sources having lower redshift quality were discarded. The catalog also contains $\sim 43,800$ photometric redshifts, obtained from the CFHT/MEGACAM deep multi-color data comprising 11 bands (Ilbert et al. 2006).

3. GEMS + GOODS-S: $~ 7000$ spectroscopic redshifts are provided from various sources, mostly obtained with ESO facilities (see Table 1 of Griffith et al. 2012). We retained redshift entries labeled with the quality flags 4 and 3 (high and good quality, respectively). The photometric redshifts $(\sim 44,200)$ for GEMS are from the COMBO-17 survey $^{18}$ (Wolf et al. 2004, 2008), whereas for the GOODS-S field we merged the relevant section of the COMBO-17 catalog with that of the latest redshift release of Dahlen et al. (2010), which comprises $\sim 32,500$ photometric redshifts derived from the 12 band $H S T / A C S$, VLT/VIMOS, VLT/ISAAC, and Spitzer photometry data.

4. GOODS-N: the ACS catalog contains 2800 spectroscopic redshifts from Barger et al. (2008) and from the GOODSN-ALL spectroscopic survey, ${ }^{19}$ which includes the Team Keck Treasury Redshift Survey (Wirth et al. 2004) and the Cowie et al. (2004) survey. As spectroscopic redshift quality metrics are not available for these data, they are all considered as reliable. As for the photometric redshifts, we merged the $\sim 6300$ catalog entries from Bundy et al. (2009; 6 bands) with the $\sim 9700$ source redshifts of Kajisawa et al. (2011; 11 bands), selected from the Subaru/MOIRCS nearinfrared data.

Given that we assembled redshift data from various sources, it is useful to review the overall photo- $z$ quality of the galaxies entering our sample through comparison with the available spec- $z$. If $\Delta z=z_{\text {spec }}-z_{\text {phot }}$, we can estimate the redshift accuracy from $\sigma_{\Delta z /\left(1+z_{\text {spec }}\right)}$, using the normalized median absolute deviation (NMAD; Hoaglin et al. 1983), defined as $1.48 \times \operatorname{median}\left\{\left|z_{\text {spec }}-z_{\text {phot }}\right| /\left(1+z_{\text {spec }}\right)\right\}$. The NMAD is directly comparable to other works, which quote the quantity rms $/(1+z)$. This dispersion estimate is robust with respect to "catastrophic errors" (i.e., objects with $\left.\left|z_{\text {spec }}-z_{\text {phot }}\right| /\left(1+z_{\text {spec }}\right)>0.15\right)$, whose percentage is denoted by $\eta$. Figure 1 (left panel) shows the comparison between $z_{\text {spec }}$ and $z_{\text {phot }}$ for the 9664 galaxies (all fields) with apparent magnitude $m_{\mathrm{AB}}<24$ and $z_{\mathrm{spec}} \leqslant 1$. We obtain an accuracy of $\sigma_{\Delta z /\left(1+z_{\text {spec }}\right)}=0.010$ for the photometric redshifts, with a percentage of "catastrophic" failures of $2.3 \%$.

For each field, all the available redshift data sets were merged together: whenever available, a "reliable" spectroscopic redshift value was used, while for the remainder of the sample, photometric redshifts were instead adopted.

\section{ANCILLARY DATA SETS}

In order to allow for a detailed characterization of the selected galaxies, and in particular to obtain galaxy mass estimates from optical-to-NIR spectral energy distribution (SED) fits to the restframe $K$-corrected magnitudes, several multi-wavelength data sets were also assembled, as detailed below.

\footnotetext{
17 http://deep.berkeley.edu/DR3/dr3.primer.html

18 http://www.mpia-hd.mpg.de/COMBO/combo_CDFSpublic.html

19 http://tkserver.keck.hawaii.edu/tksurvey/data_products/goods_desc.html
} 



Figure 1. Left panel: comparison between $z_{\text {phot }}$ and $z_{\text {spec }}$ for our final galaxy sample (all fields) with MAG_BEST_HI $<24$. The dotted and dashed lines are for $z_{\text {phot }}=z_{\text {spec }} \pm 0.15\left(1+z_{\text {spec }}\right)$ and $z_{\text {phot }}=z_{\text {spec }} \pm 0.05\left(1+z_{\text {spec }}\right)$, respectively. The $1 \sigma$ dispersion and the fraction of catastrophic failures $\eta$ are listed in the top left corner of the right panel. Right panel: $\Delta z /\left(1+z_{\text {spec }}\right)$ distribution. The dashed line is a Gaussian distribution with $\sigma=0.010$.

(A color version of this figure is available in the online journal.)

\subsection{Optical, UV, and Near-infrared Surveys}

\subsubsection{COSMOS}

The COSMOS photometric data were taken from the publicly available COSMOS Intermediate and Broad-Band Photometric catalog, ${ }^{20}$ the UltraVISTA Survey Data Release 1 (McCracken et al. 2012), ${ }^{21}$ and Spitzer/IRAC bands 1-2 (3.6 and $\left.4.5 \mu \mathrm{m}\right)$ data from the S-COSMOS public catalog 22 (Sanders et al. 2007).

The COSMOS catalog comprises: ultraviolet data from GALEX and CFHT/ $u^{*}$-band imaging; in the optical, the ACS/ $I_{\mathrm{F} 814 \mathrm{~W}}$-band data and the COSMOS-21 survey on Subaru (Taniguchi et al. 2007), including 6 broad-band $\left(B_{J}, V_{J}, g^{+}, r^{+}\right.$, $i^{+}, z^{+}$), 12 medium-band (IA427, IA464, IA484, IA505, IA527, IA574, IA624, IA679, IA709, IA738, IA767, IA827), and 2 narrow-band (NB711, NB816) filters. Total magnitudes were obtained from the corresponding aperture values by applying the recommended aperture corrections. Only reliable photometric measurements were considered, i.e., data with bad pixel or band mask flags different than zero were discarded. Recommended photometric offsets (Ilbert et al. 2009) and galactic extinction corrections (Capak et al. 2007) were also applied.

In the near-infrared, we used the VIRCAM/Y-, $\mathrm{H}_{-}, \mathrm{J}-$, and $K_{s}$-bands imaging data from the UltraVISTA survey and Spitzer/IRAC bands 1-2 (3.6 and $4.5 \mu \mathrm{m})$ data from the S-COSMOS. SEXTRACTOR "auto" (or Kron-like) magnitudes were used for near-infrared UltraVISTA data, whereas IRAC channels 1-2 total magnitudes were calculated from the aperture-corrected fluxes of the S-COSMOS data release. Unreliable flux entries (e.g., close to bright stars or close to the image margins) were discarded.

\footnotetext{
20 http://irsa.ipac.caltech.edu/data/COSMOS/gator_docs/cosmos_ib_ colDescriptions.html

21 http://www.eso.org/sci/observing/phase3/data_releases/ultravista_dr1.html

22 http://irsa.ipac.caltech.edu/data/COSMOS/gator_docs/scosmos_irac_ colDescriptions.html
}

\subsubsection{AEGIS}

For the AEGIS field, the following data were considered: GALEX far-ultraviolet and near-ultraviolet data from the EGS multi-wavelength data $\operatorname{set}^{23}$ (Davis et al. 2007), HST/ACS $V_{\mathrm{F} 606 \mathrm{~W}^{-}}$and $I_{\mathrm{F} 814 \mathrm{~W}}$-band photometry ${ }^{24}$ (Lotz et al. 2008), CFH12K $B-, R$-, and $I$-band photometry ${ }^{25}$ (Coil et al. 2004), CFHT Megacam $u-, g_{-}, r-, i-$, and $z$-band photometry ${ }^{26}$ (Gwyn 2008), Palomar/WIRC $J$ - and $K_{s}$-band data ${ }^{27}$ (Bundy et al. 2006), and Spitzer/IRAC bands 1-2 (3.6 and $4.5 \mu \mathrm{m})$ data $^{28}$ (Barmby et al. 2008). Together, these data sets provide up to 15 bands for spectral energy distribution analysis.

\subsubsection{GEMS}

GEMS sources were analyzed using the optical multi-band photometry from the COMBO-17 survey (Wolf et al. 2004, 2008), which provides observations in 17 optical filters in the 365-914 nm wavelength range, obtained with the Wide Field Imager at the MPG/ESO $2.2 \mathrm{~m}$ telescope. ${ }^{29}$ Aperture-corrected photon fluxes were considered in the analysis.

\subsubsection{GOODS-S}

For the GOODS-S sources we used a 12 band selection of the high quality multi-wavelength data of GOODS-MUSIC ${ }^{30}$ (Multi-wavelength Southern Infrared catalog; Grazian et al. 2006). This includes $U$-band data from the $2.2 \mathrm{ESO}$ and VLT/VIMOS, $H S T /$ ACS $B_{435}, V_{606}, i_{775}$, and $z_{850}$ filters, the $J H K_{s}$ VLT data, as well as the 3.6 and $4.5 \mu \mathrm{m}$ Spitzer data.

\footnotetext{
23 http://www.galex.caltech.edu/researcher/techdoc-ch5a.html

$24 \mathrm{http}: / /$ aegis.ucolick.org/acs_datasets.html

25 http://deep.berkeley.edu/DR1/dr1.primer.html

${ }^{26}$ http://www3.cadc-ccda.hia-iha.nrc-cnrc.gc.ca/community/CFHTLS-SG/ docs/cfhtls.html

${ }^{27} \mathrm{http}: / /$ www.astro.caltech.edu/AEGIS/

28 http://www.cfa.harvard.edu/irac/egs/

${ }^{29}$ See http://www.mpia.de/COMBO/combo_filters.html for a comprehensive description of the filter set.

30 http://cdsarc.u-strasbg.fr/viz-bin/qcat?J/A+A/449/951
} 
MUSIC provides SEXTRACTOR “auto" (or Kron) magnitudes for the ACS images, whereas for ground-based and Spitzer data, reliable estimates of the total magnitudes were retrieved through PSF-matching.

\subsubsection{GOODS-N}

Optical photometric data for the GOODS-N field (Giavalisco et al. 2004) were taken from the GOODS r2.0 $z^{31}$ ACS multiband source catalog and, at longer wavelengths, from the WIRCam ultra-deep catalog of GOODS-N (Wang et al. 2010). We thus considered a 7 band catalog, which comprises the $B_{\mathrm{F} 435 \mathrm{~W}^{-}}, V_{\mathrm{F} 06 \mathrm{~W}^{-}}, i_{\mathrm{F} 75 \mathrm{~W}^{-}}$, and $z_{\mathrm{F} 850 \mathrm{LP}}$-filter data, plus $K_{s^{-}}$and IRAC 1-2 bands (3.6 and $4.5 \mu \mathrm{m})$. SExTRACTOR "best" and "auto" magnitudes were chosen for the visible bands and $K_{s}$ data, respectively. The $3.6 \mu \mathrm{m}$ and $4.5 \mu \mathrm{m}$ fluxes derived by Wang et al. (2010) were instead obtained by a deconvolution procedure based on a $K_{s}$ image prior, which is expected to deliver highly accurate near-infrared and mid-infrared colors.

\subsection{Mid-IR Surveys}

Spitzer Space Telescope mid-infrared data were used to evaluate star-forming rates (SFRs). MIPS $24 \mu \mathrm{m}$ public catalogs are available for COSMOS (S-COSMOS GO2 and GO3 releases ${ }^{32}$; Frayer et al. 2009) and for AEGIS (Barro et al. 2011), while for GOODS-S/GEMS fields we used a catalog of sources extracted from FIDEL 24 image (v0.5) of the Extended CDFS (6 arcsec PSF matched photometry; X. Z. Zheng, in preparation).

\subsection{X-Ray Catalogs}

X-ray surveys have targeted areas within the fields presented in our study. Given the relevance of those data for the detection of AGN, we have also assembled the available public data sets existing in these regions, namely:

1. The COSMOS-Chandra catalog (C-COSMOS, Elvis et al. 2009), and the AEGIS catalog in the Chandra field (AEGIS-X, Laird et al. 2009), with 1761 and 1325 X-ray point sources detected, respectively.

2. The XMM-Newton-COSMOS catalog (XMM-COSMOS; Cappelluti et al. 2009; Brusa et al. 2010), which detected 1887 unique sources over an area of $2 \mathrm{deg}^{2}$.

3. The catalogs of the 2 Ms Chandra Deep Field-North (CDF-N; Alexander et al. 2003), with a total of 503 detections, the 4 Ms Chandra Deep Field-South (CDF-S; Xue et al. 2011), which contains 776 sources, and also the wider coverage $1 \mathrm{Ms}$ Extended Chandra Deep Field-South (E-CDF-S; Lehmer et al. 2005), with 795 sources.

\section{THE FINAL CATALOG OF BULGELESS GALAXIES}

After having assembled the various data sets, we have obtained a homogeneous sample of rest-frame ultraviolet to NIR magnitudes for all galaxies by using the publicly available kcorrect code (version v4_2) ${ }^{33}$ described in Blanton \& Roweis (2007). kcorrect is designed to extract the most physically realisable SED by using linear combinations of five SEDs that are characteristic of physical states of galaxies, from intense starburst to quiescent objects. Also, the implementation of the code makes it particularly suitable for modeling optical and

\footnotetext{
31 http://archive.stsci.edu/pub/hlsp/goods/v2/h_goods_v2.0_rdm.html

$32 \mathrm{http}: / /$ irsa.ipac.caltech.edu/data/COSMOS/gator_docs/scosmos_mips 24_go3_colDescriptions.html

33 Available at http://howdy.physics.nyu.edu/index.php/Kcorrect.
}

Table 2

Catalog Selection Statistics Split by Field $(0.4 \leqslant z \leqslant 1.0)$

\begin{tabular}{lrrrcr}
\hline \hline Field & Total & spec- $z$ & $n \leqslant 1.5$ & $1.5<n \leqslant 3.0$ & $n>3.0$ \\
\hline COSMOS & 31714 & 3116 & 14139 & 7259 & 10316 \\
AEGIS & 2848 & 1451 & 1588 & 576 & 684 \\
GEMS & 3595 & 1382 & 2267 & 793 & 535 \\
GOODS-S & 852 & 524 & 482 & 199 & 171 \\
GOODS-N & 843 & 648 & 749 & 74 & 20 \\
Total & 39852 & 7121 & 19225 & 8901 & 11726 \\
\hline
\end{tabular}

near-infrared observations in the redshift range $0<z<1.0$. The galaxy templates are based on the Bruzual \& Charlot (2003) stellar evolution synthesis models and, together with rest-frame magnitudes, this tool also provides estimates of the stellar mass-to-light ratio (see Section 4.2). kcorrect seeks the best fit to each source photometry using a linear combination of five spectral templates $F(\lambda)$, which are, in turn, derived by non-negative matrix factorization (NMF) of 485 basis templates spanning grids of metallicities $(Z=0.0001 \sim 0.05)$ and ages ( 1 Myr $\sim 13.75 \mathrm{Gyr}$ ), with three choices of dust models (no extinction, Milky-Way-, and Small-MagellanicCloud-type extinction). Thirty-five templates of line emission from the ionized gas (MAPPING-III, Kewley et al. 2001) are also included in the model. $K$-corrections were calculated for all entries of our sample (irrespective of $n$ ), which have both $z \leqslant 1.0$ and at least five photometric detections.

We thus obtained rest-frame absolute magnitudes for all objects in our sample. For the objects lacking one or more photometric data-points, rest-frame absolute quantities have been derived directly from the fitted SEDs. We have then generated a homogeneous 11 filter data set comprising: GALEX far-ultraviolet, near-ultraviolet, Johnson's $U, B, V, I$, SDSS $g, r$, $z$, Vircam ${ }^{34} J$ and $K_{s}$ bands. The reduced $\chi^{2}$ of each SED fit is retained in the final catalog.

A definition of $n \leqslant 1.5$ was applied to select galaxies with little or no contribution of the bulge to the light profile (bulgeless galaxies; Gadotti 2009). As control samples we also selected galaxies with different morphological properties, adopting the following criteria: $1.5<n \leqslant 3.0$ corresponding to disk galaxies with an increasingly prominent bulge component, and $n>3$ for bulge-dominated galaxies. These samples were used to test the morphological evolutionary scenarios discussed in recent literature (e.g., Pannella et al. 2009; Oesch et al. 2010). A summary of the final sample composition is reported in Table 2. The redshift distribution of the galaxy sample including all the morphological types is shown in the left panel of Figure 2. In the right panel we show the distribution of galaxy stellar masses as a function of redshift.

In summary, we have created a magnitude-limited $\left(m_{\mathrm{AB}}<\right.$ 24 , in the reddest available $H S T$ band, $\sim 7750$ to $8500 \AA$ ), low-inclination, point-source free, $K$-corrected galaxy catalog for five fields (COSMOS, AEGIS, GOODS-S, GEMS, and GOODS-N) with robust intermediate $(0.4 \leqslant z \leqslant 1.0)$ redshift measurements (photometric and spectroscopic) and reliable morphological data, containing a total of 19,225 bulgeless galaxies out of 39,852 sources. A conservative estimate for the mass completeness of this catalog, considering the mass of the model SED used by kcorrect with the highest

\footnotetext{
34 VIRCAM is a wide field imager for survey mounted on the VISTA telescope. See http://www.eso.org/sci/facilities/paranal/instruments/vircam/inst/ for more information on the filter set.
} 

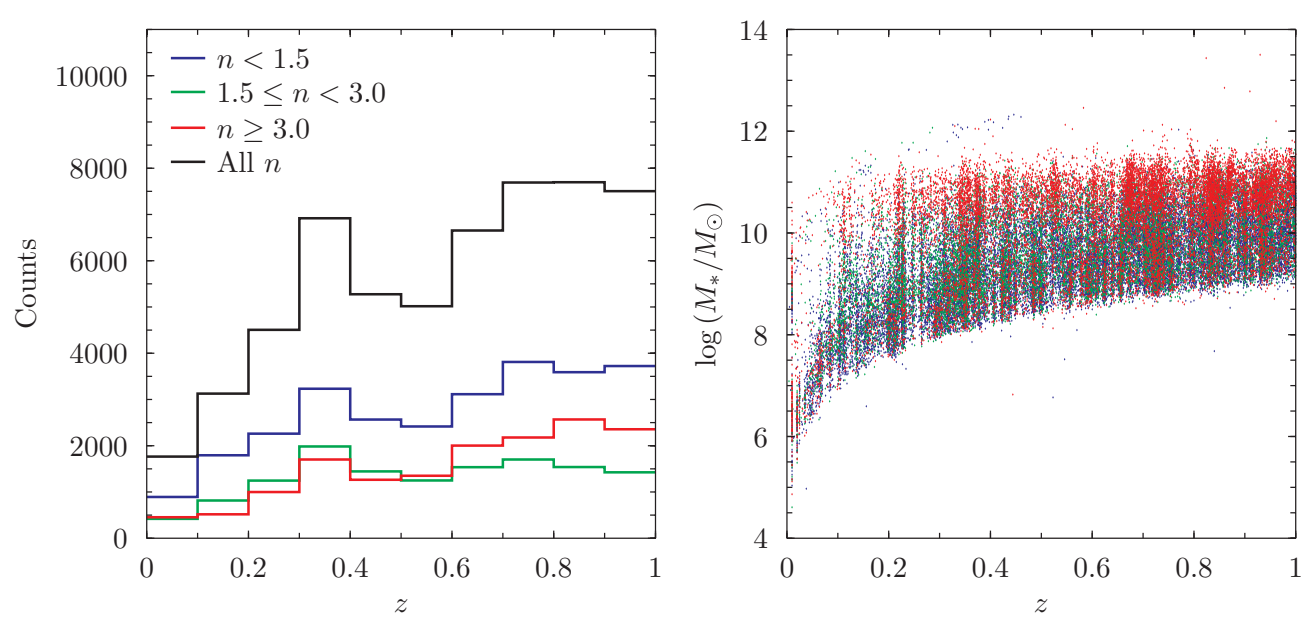

Figure 2. Left panel: redshift distribution for the 39,852 galaxies belonging to the morphological catalog defined in the present work. Different Sérsic index $n$ ranges are indicated by colors: blue $n<1.5$, green $1.5 \leqslant n<3.0$, and red $n \geqslant 3.0$. Right panel: distribution of galaxy stellar masses derived using kcorrect (see Section 4) as a function of redshift. Blue, green, and red symbols label the three morphological types as in the left panel.

(A color version of this figure is available in the online journal.)

mass-to-light ratio, results in a minimum measurable stellar mass of $1.0 \times 10^{9} M_{\odot}$ at $z=0.4$, and $4.3 \times 10^{10} M_{\odot}$ at $z=1$.

As mentioned in Section 2.1, we adopted a filtering criterion to exclude point sources from the final galaxy sample in order to reduce the contamination produced by spurious morphological classification. This led us to reject 9049 sources in the redshift interval $0.4 \leqslant z \leqslant 1.0$, corresponding to $23 \%$ of the sample size. Due to the lack of reliable morphology, these missing objects might potentially alter the content of the catalog in a not well predictable way. We thus inspected the point-source sample to asses its nature and to estimate the magnitude of the potential biases produced in the galaxy-type class analysis. Figure 3 shows the $B-z$ versus $z-K$ color-color plot of 9979 compact source at $z \leqslant 1.0$. In the $B z K$ plot, stars are segregated in the region defined by the relation $(z-K)<0.3(B-z)-0.5$ (e.g., Daddi et al. 2004) and can be efficiently separated from galaxies. Blue symbols in Figure 3 label the 7015 stars that can be identified using this method, whereas the black dots located in the upper part of the $B z K$ plane (2964 objects, 30\%) are likely to be small size galaxies that we ultimately discarded from our morphological catalog. This test indicates that the adopted pointsource rejection criterion led at most to a 7\% decrement of the magnitude-limited galaxy sample in the interval $0.4 \leqslant z \leqslant 1.0$. The neglected population shows only a mild redshift dependence (see the inset of Figure 3), thus we are thus confident that the main results of this work are not significantly affected.

As an example of the catalog content, we show in Figure 4 sample cutouts of selected bulgeless galaxies spanning the full redshift range and the $6 \times 10^{9}<M / M_{\odot}<2 \times 10^{11}$ stellar mass interval. The catalog of the absolute magnitudes, including morphological information and stellar mass estimates analyzed in this work is made publicly accessible at the following URL: http://www.oal.ul.pt/ jafonso/Bulgeless

\subsection{Reliability of the Morphological Classification}

One of the goals of this work is the selection of a reliable sample of intermediate redshift galaxies having very little or no contribution from a bulge to their light profile. The sample was homogeneously assembled from the imaging data of four different $H S T /$ ACS fields, and the morphological classification relied entirely on an automated single-component parametric-

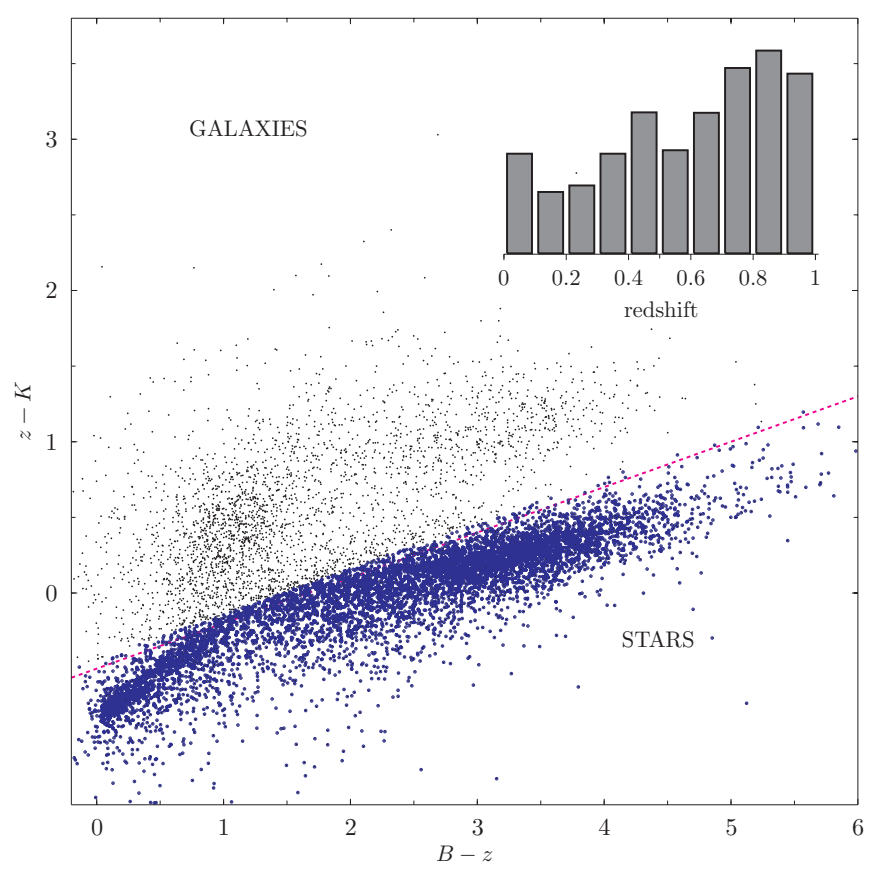

Figure 3. $(z-K)$ vs. $(B-z)$ color-color diagram for the compact objects selected as described in Section 2.1 and removed from the final morphological catalog. The diagonal dotted line defines the region $(z-K)<0.3(B-z)-0.5$, which is preferentially occupied by stars (Daddi et al. 2004). Stars selected using this criterion are labeled with blue asterisks. Black dots located above the dotted line identify the point-like galaxies having little or no morphological information and that are rejected from the final sample. The inset shows the redshift distribution of this compact galaxy sample.

(A color version of this figure is available in the online journal.)

fit method. Due to the large content of the sample $(\sim 40,000$ objects), no extensive visual confirmation of the estimated morphology is feasible. Nevertheless, it is important to assess the reliability of the bulgeless classification of our sample through comparison with some independent analysis and a careful consideration of the different sources of uncertainty.

First of all, to assess the wavelength dependence of galaxy morphology, we cross-checked our ACS-GC GALFIT classification with the results of a similar structural analysis performed by Wuyts et al. (2011) on GOODS-S sources spanning 


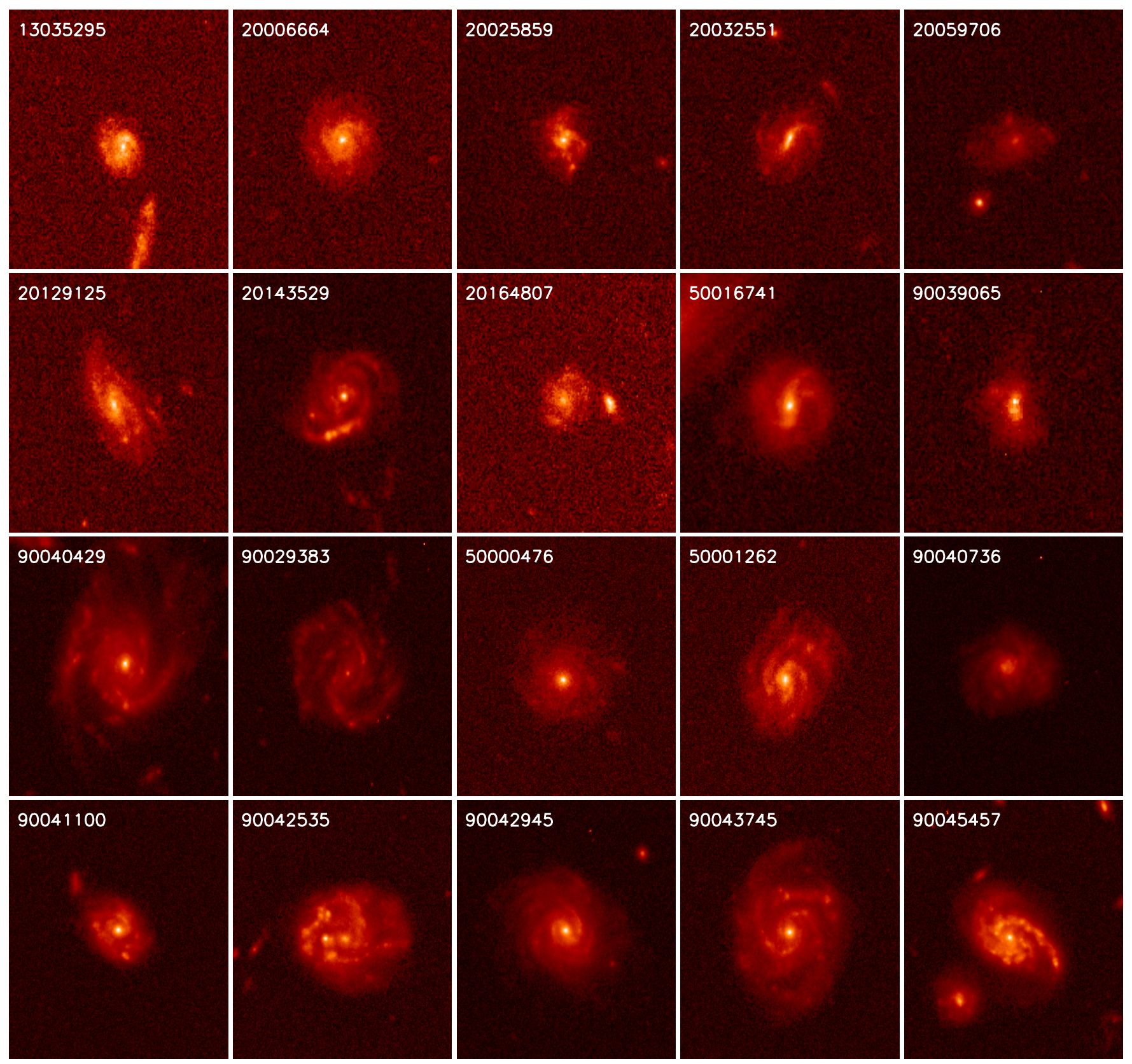

Figure 4. HST/ACS sample images of 20 selected bulgeless galaxies spanning the $0.4 \leqslant z \leqslant 1.0$ redshift range and $6 \times 10^{9}<M / M_{\odot}<2 \times 10^{11}$ stellar mass interval. The first two upper rows show $160 \times 160$ pixel cutouts, while the third and fourth rows show $256 \times 256$ pixel cutouts.

(A color version of this figure is available in the online journal.)

$0.1 \lesssim z \lesssim 2.5$ using CANDELS $H_{160}$ data. The results of comparison are shown in Figure 5. In spite of the large rest-frame wavelength difference probed by the two surveys, the comparison between the two sets of Sérsic $n$ index is fair, and the systematic discrepancies are limited to a few percent over the entire magnitude interval covered by our sample.

Second, we evaluated the overall reliability of our GALFITbased morphologies through the comparison to a method based on different classification criteria. In Scarlata et al. (2007), a sample of 56,000 galaxies in COSMOS was the subject of a detailed morphological study, adopting the Zurich Estimator of Structural Types (ZEST), a sophisticated algorithm that uses a combination of non-parametric and parametric quantification of galaxy structure. Its classification scheme comprises type 1 (spheroids with no visible disk), type 2 (disk galaxies), and type 3 (irregular) galaxies. A "bulgeness" index is also provided for type 2 disk galaxies, which coarsely correlates with bulgeto-disk ratio: it ranges from pure disk galaxies (type 2.3) to bulge-dominated disks (type 2.0). Since their analysis was also performed on the ACS F814W COSMOS images, the comparison with our Sérsic-index based morphological classification presented here is straightforward.

We cross-matched the publicly available Zurich morphology catalog $^{35}$ with our COSMOS sub-sample, obtaining 30,104 galaxies with both a GALFIT-derived Sérsic index and a ZEST classification. The numerical results of this comparison split by ZEST classes are shown in Table 3 . We found that 13,073 out of 13,680 galaxies $(96 \%)$ of our bulgeless $(n \leqslant 1.5)$ sample

\footnotetext{
35 http://irsa.ipac.caltech.edu/data/COSMOS/datasets.html
} 


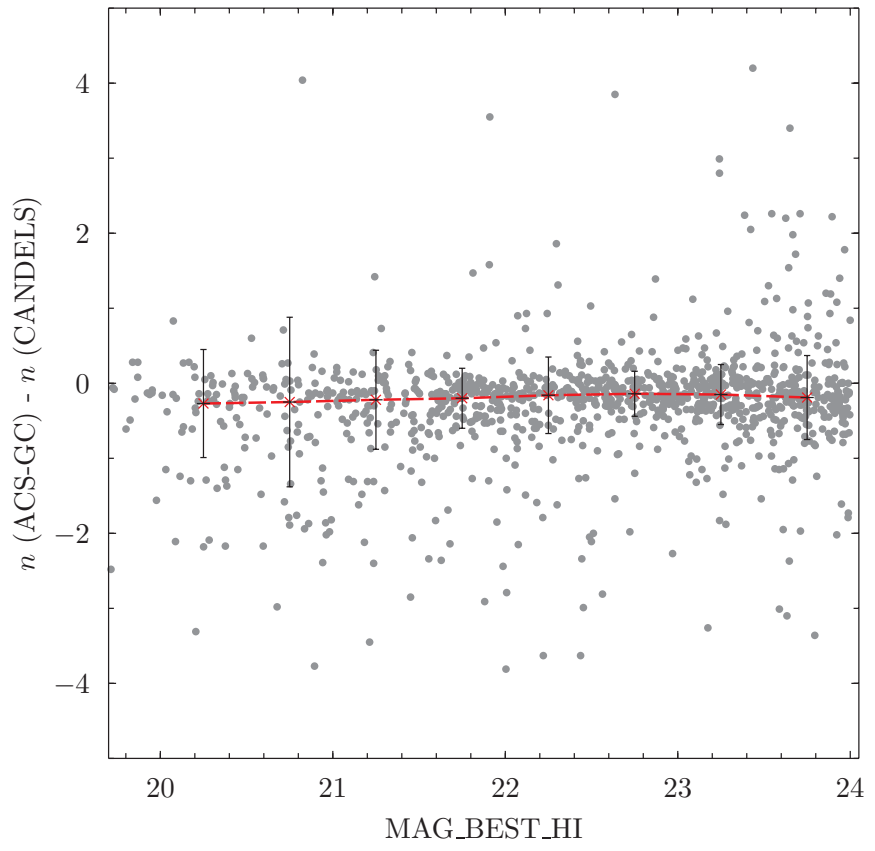

Figure 5. Comparison between GALFIT generated Sérsic index $n$ in GOODS-S sources using ACS-GC $z_{\text {F850LP }}$ and CANDELS $H_{160}$ bands as a function of the apparent $z$ magnitude (all morphological types). Gray dots indicate $\Delta n$ deviations. The red line illustrates the trend of the median taken over $0.5 \mathrm{mag}$ bins. Error bars mark the central 50th percentile.

(A color version of this figure is available in the online journal.)

are classified as having little or no bulge contribution in ZEST (types 2.2, 2.3, and 3.0), $570(4 \%)$ are classified as intermediate disk galaxies (type 2.1 ), whereas a mere 37 objects $(0.2 \%)$ are considered as bulge-dominated in ZEST (type 1.0 and 2.0). This comparison indicates that our bulgeless sample might have a $5 \%$ contamination by misclassified bulge-dominated objects. This error should be summed in quadrature to the uncertainty due to the point-source rejection $(\sim 7 \%$ see Section 4$)$ to yield a conservative overall uncertainty of $8 \%$ in the morphological classification of the bulgeless sample.
Table 3

Morphological Classification Comparison of the COSMOS Sources Using ZEST (Scarlata et al. 2007) and GALFIT (This Work)

\begin{tabular}{lrcr}
\hline \hline ZEST Type & $n \leqslant 1.5$ & $1.5<n \leqslant 3.0$ & $n>3.0$ \\
\hline 1.0 & 13 & 130 & 2596 \\
2.0 & 24 & 158 & 1966 \\
2.1 & 570 & 2892 & 2760 \\
2.2 & 4162 & 2530 & 1082 \\
2.3 & 7115 & 723 & 698 \\
3.0 & 1796 & 497 & 392 \\
Total & 13680 & 6930 & 9494 \\
\hline
\end{tabular}

Not surprisingly, the agreement between our "bulgy" defined sample and the ZEST spheroidal-type classification is only moderate. ZEST type 1.0 and 2.0 collect 4562 out of 9494 objects corresponding to $48 \%$, while 2760 further galaxies (29\%) are classifies as intermediate type 2.1 (disks with prominent bulges). It should be noted that the ZEST classes 1.0 and 2.0 mostly include classical spheroidal objects whose surface brightness is best reproduced by De Vaucouleurs' profile with $n=4$. On the other hand, the $n \geqslant 3$ criterion we adopted to define the "bulgy" sample is broader and it is expected to include more objects having a significant disk component. Given that this study is focused on bulgeless object, this comparison provides a confirmation of the methodology used, i.e., that the Sérsic index criterion has a good reliability in selecting galaxies with little bulge contribution.

\subsection{Accuracy of Mass Estimates}

The overall view in the literature (e.g., Bell \& de Jong 2001; Drory et al. 2004; Muzzin et al. 2009; Hainline et al. 2011) is that uncertainties in the various assumptions (i.e., redshift, initial mass function, stellar population synthesis (SPS) model, star-formation history, dust extinction, etc.) imply an overall conservative uncertainty of up to a $\sim 2-3$ factor $(\sim 0.2-0.5$ dex $)$ for each individual mass estimate. Therefore, we compared our kcorrect results with those independently presented by Pannella et al. (2009) for COSMOS and by the FIREWORKS team (Marchesini et al. 2009) for GOODS-S. The comparison of the stellar masses is shown in Figure 6. All three methods employ
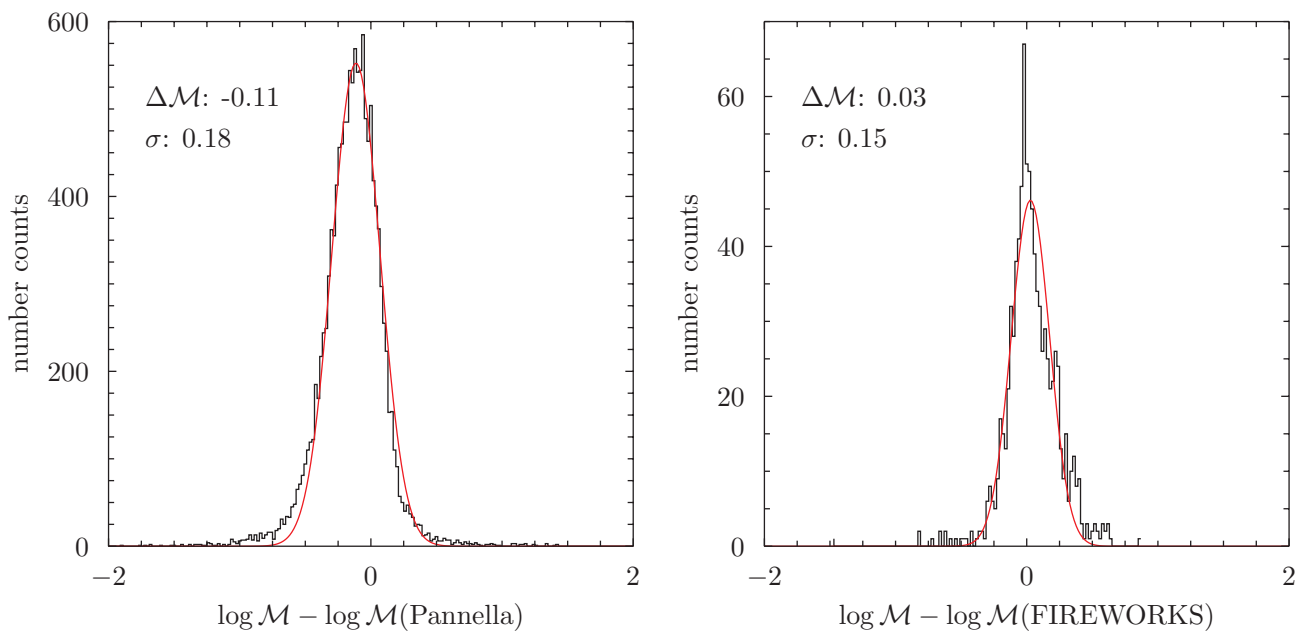

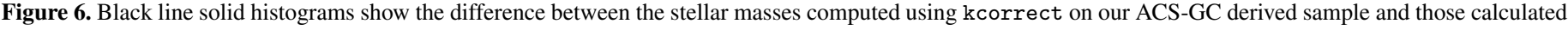

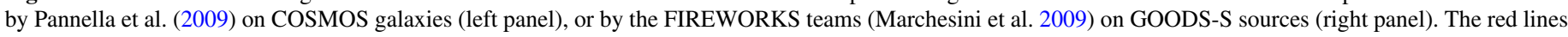
are Gaussian distributions with $\sigma=0.18$ (left panel) and $\sigma=0.15$ (right panel).

(A color version of this figure is available in the online journal.) 


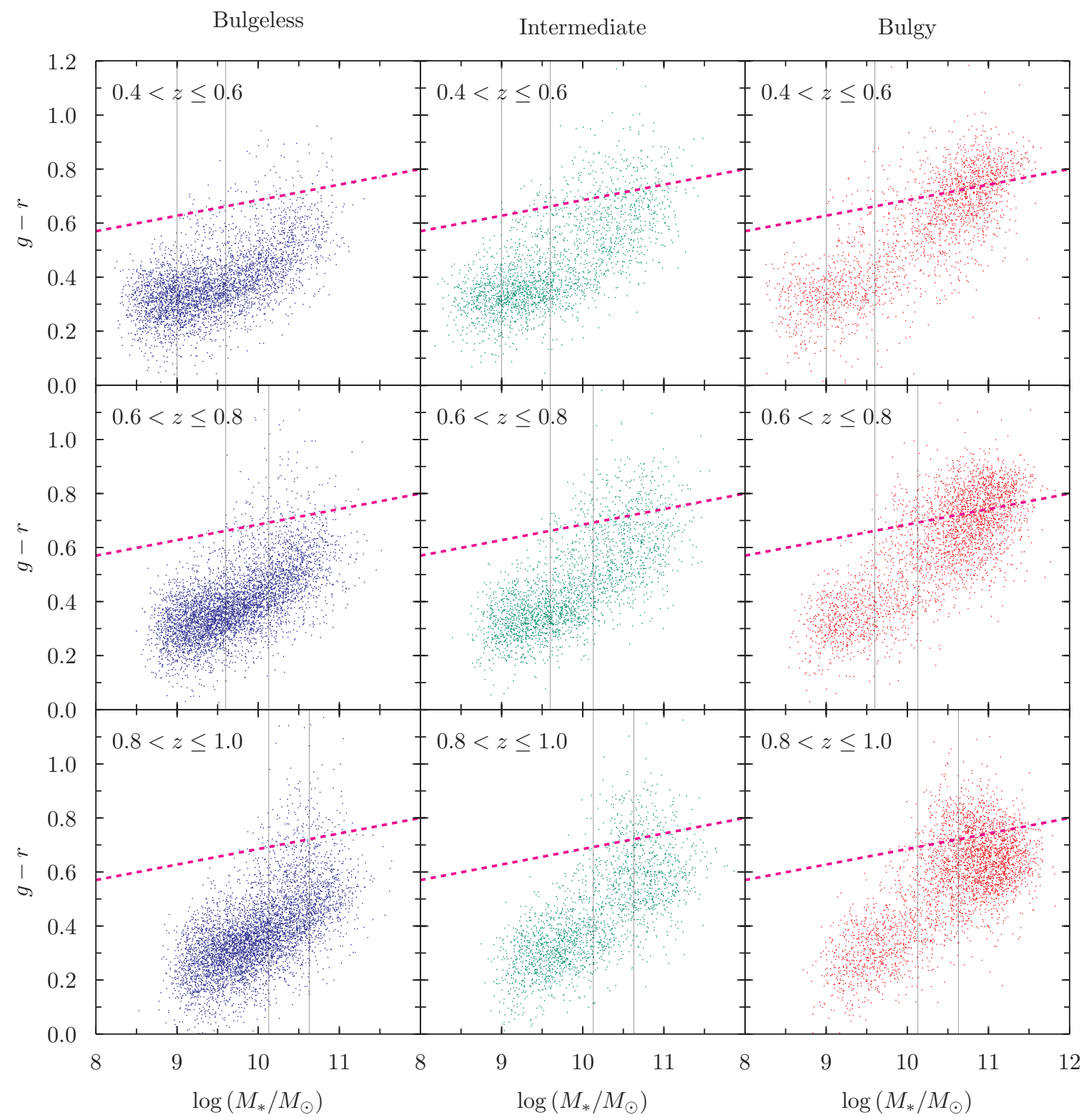

Figure 7. Rest-frame $g-r$ color-mass distribution for the final, low-inclination, magnitude-limited catalog. The galaxies are separated into three sub-samples: those lacking significant bulge (Sérsic index $n<1.5$; left), those with an intermediate disk-to-bulge ratio $(1.5 \leqslant n<3.0$; central), and those which are bulge-dominated $(n \geqslant 3.0$; right. Also, each sub-sample has been divided in three redshift bins: $0.4<z<0.6$ (top), $0.6<z<0.8$ (middle), and $0.8<z<1.0$ (bottom). The dashed purple lines indicates the red sequence locus (see text), the two vertical dotted lines show the mass completeness limit at the upper and lower edges of each redshift bin.

(A color version of this figure is available in the online journal.)

the Bruzual \& Charlot (2003) SPS model. The FIREWORKS mass determinations closely match our kcorrect-derived values, whereas those of Pannella et al. (2009) are 0.11 dex larger, on average, which is within the range of uncertainties referred by these authors. In both cases, the scatter is less than 0.2 dex, still within the overall uncertainty referred above.

\section{BULGELESS GALAXY PROPERTIES}

\subsection{Color Evolution at Intermediate Redshift}

Figure 7 shows the rest-frame $g-r$ color as a function of mass, redshift, and morphological classification. Three redshift ranges are considered (row-wise): $0.4<z \leqslant 0.6,0.6<z \leqslant$ 0.8 , and $0.8<z \leqslant 1.0$. The oblique dashed line represents the red sequence, defined as by Bell (2008) in the SDSS sample: $(g-r)>0.57+0.0575 \log \left(M_{*} / 10^{8} M_{\odot}\right)$.

The left-hand panels show the color-mass properties of the bulgeless sample, which is the goal of the present investigation. The bulgeless population at all redshifts exhibits a striking segregation at bluer colors compared to the other morphological types, with few objects extending beyond the red sequence line. The properties of the bulgeless sample are discussed in more details in Sections 5.2 and 5.3.

Bulge-dominated galaxies ( $n \geqslant 3.0$, right panels of Figure 7), are mostly located in two regions in the color-mass diagram, with a clear segregation in mass. Massive objects $\left(M_{*}>\right.$ $5 \times 10^{10} M_{\odot}$ ) are located on the red sequence at all redshifts. They do not show an appreciable evolution in mass (the fraction of galaxies with $\log \left(M_{\star} / M_{\odot}\right)>10.5$ remains approximately 




Figure 8. Number densities as a function of redshift and morphology for galaxy masses above the completeness limit. Symbols are colored according to the morphological classification (blue: bulgeless; green, intermediate; red: bulgy). The error bars were calculated by adding in quadrature the following contributions: (1) cosmic variance (Moster et al. 2011), (2) the square root of the number of galaxies per redshift bin, and (3) the 0.5 dex error estimated in the stellar mass determination.

(A color version of this figure is available in the online journal.)

constant at $\sim 0.9$ irrespective of redshift bin) and move slightly toward redder colors with cosmic time, an effect of the ageing of the stellar population. On the lower mass end of the distribution $\left(M_{*}<10^{10} M_{\odot}\right)$, there exists a less numerous, but still appreciable, population of bulge-dominated galaxies, reaching the blue cloud. These could be low-mass bulge-dominated galaxies hosted in low-density groups, where they are able to sustain appreciable star formation, possibly via "wet merger" events. Indeed, early-type galaxies with young stellar populations at low $z$ are predominantly low-velocity dispersion systems and tend to live in lower density regions (Suh et al. 2010; Thomas et al. 2010). Moreover, some Blue Compact Dwarfs, particularly those classified as nE types in Loose \& Thuan (1986a), also show surface brightness profiles fitted by a de Vaucouleurs $R^{(1 / 4)}$ profile (Loose \& Thuan 1986b; Kunth et al. 1988; Cairós et al. 2001). Assessing the proper nature of these objects is beyond the scope of this study; however, we refer to Pannella et al. (2009) for a more detailed analysis of the environment of blue early-type galaxies at intermediate redshift.

The intermediate-type population (with $1.5 \leqslant n<3.0$, central panels of Figure 7) is mainly composed of disk or spiral galaxies having a prominent bulge. In the high-redshift end of the diagram they extend from the red sequence to the blue cloud region, whereas at lower- $z$ they are more concentrated in the blue/low-mass region. This agrees with the conclusions of previous studies on galaxy evolution (Pannella et al. 2009; Oesch et al. 2010), although these authors have used a different morphological classification scheme.

Concerning the bulgeless galaxies, if we focus on the highmass end (e.g., for $\log \left(M_{\star} / M_{\odot}\right)>10.5$, where our sample is complete at all $z$ ), one can see a decreasing number of objects from $z \sim 1$ to the lowest redshift bin. To take properly into account the survey volume effects, we evaluated the number density evolution of each morphological type for stellar masses $\log \left(M_{\star} / M_{\odot}\right)>10.5$. The result is illustrated in Figure 8, where it is clear that for the high-mass range considered, bulge-dominated systems constitute the majority of the galaxy population in the redshift range $0.4 \leqslant z \leqslant 1.0$.

Looking at the evolution of the different morphological types, one can indeed see that, contrary to the number densities of massive bulgy and intermediate galaxies, which do not evolve significantly between $z \sim 0.4$ and $z \sim 1$, the bulgeless galaxy population shows a significant decrease in their number density as cosmic time increases. The fact that early-type objects, in the selected mass range, show little or no evolution is in agreement with the most massive E/S0 galaxies being already in place at $z \sim 1$ and evolve only weakly since then (Collins et al. 2009). On the other hand, the observed disappearance of massive bulgeless galaxies at later cosmic times necessarily implies a process that accompanies the growth of their stellar masses and tends to displace them to an early-type morphological bin through the formation of a classical bulges.

Oesch et al. (2010) attempted to quantify this observed trend assuming the morphological transformation of galaxies as due exclusively to merger events. Their simulations were unable to reasonably fit the observed data for all morphological types simultaneously, even including small accretion events in the model. Most notably, all the models overpredict the mass fraction of bulgeless disk galaxies as cosmic time proceeds. Thus, it is likely that these objects undergo a bulge-building process driven by disk dynamical instability, possibly triggered by secular accretion activity (Kormendy \& Kennicutt 2004).

As a final remark, we remind that the possible bias introduced by the point-source rejection is estimated to be weak and scarcely redshift dependent (see discussion in Section 4), so that its effects are expected to be within the error bars shown in Figure 8.

\subsection{Red Bulgeless Galaxies: Quiescent or Dusty?}

Recent studies (Bell 2008; Bell et al. 2012) demonstrated that the Sérsic index, among other galaxy quantities, correlates best with the lack of star-formation activity. At $z<0.05$, large Sersíc indices correlate extremely well with quiescence, as shown by Bell (2008) using a SDSS sample. It is argued that "genuine" bulgeless quiescent galaxies could be either (1) satellite galaxies in high-mass halos, whose gas is stripped in a deep potential well, or (2) possibly a result of incorrect morphological classification, given that many of them show a hint of a bulge once visual examination is performed. Quenched bulgeless galaxies are rather rare up to high redshift $(z \sim 2.2$; Bell et al. 2012), and only a few pure disk, quiescent systems are reported in the literature at $z \gtrsim 1$ (e.g., McGrath et al. 2008; van der Wel et al. 2011; Bundy et al. 2010).

As discussed in Section 5.1, it is known that the red sequence is mostly composed of galaxies with elliptical morphology and prominent bulges (e.g., Cassata et al. 2007). Nonetheless, it also hosts a small fraction of dusty, late-type, reddened starforming objects (Williams et al. 2009) and Spi/Irr galaxies, with quenched star-formation (see, e.g., Bell et al. 2012 and references therein). Indeed, Figure 9 (left panel) shows that a significant fraction of the bulgeless population is located in the red sequence region of the color-mass diagram. Finding red bulgeless galaxies gives evidence for an intriguing population of late-type objects - their optically red nature may result from substantial dust obscuration but it could also indicate that they have ceased forming stars, thus revealing a mechanism that induces quiescence without significantly altering the morphology.

A way to break the degeneracy between dusty star-forming and red quiescent galaxies is the use of rest-frame nearinfrared-optical colors $U-V / V-J$ (Wuyts et al. 2007; Williams et al. 2009). Essentially, the $U-V$ color provides a proxy for unobscured star-formation activity, whereas $V-J$ helps in highlighting dust-free quiescent galaxies that exhibit 

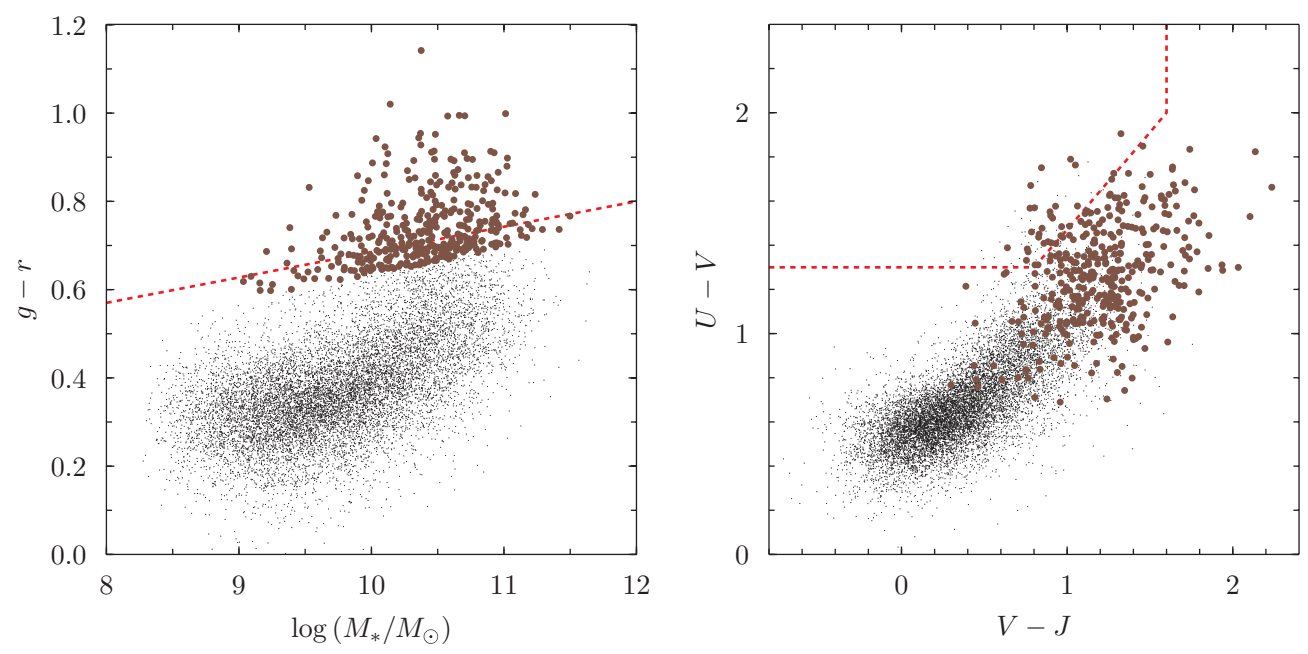

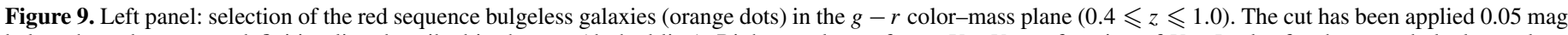

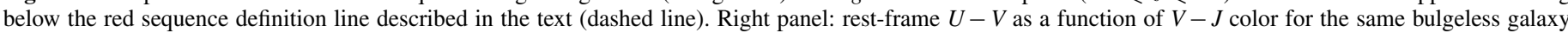


lines. According to this plot, the vast majority of red bulgeless galaxies are red because of the dust and not because of an older stellar population.

(A color version of this figure is available in the online journal.)

a bluer color than dusty objects, and thus occupy a different locus in the $U V J$ plane. The relevant plot is shown in the right panel of Figure 9. The superimposed dashed lines are the redshiftdependent selection criteria for quiescent galaxies, described by Williams et al. (2009). To discriminate between quiescency and dust obscuration, we highlighted in the $U V J$ plane the subsample of red bulgeless lying down to 0.05 mag below the red sequence definition line indicated in Section 5.1. The plot indicates that most of the bulgeless galaxies that are red in the $g-r /$ mass diagram owe their optical color to dust instead of an old stellar population, with only a few objects located in the region of the diagram corresponding to quiescent galaxies.

\subsection{Massive Bulgeless Galaxies}

Figures 7 and 8 show that our sample includes a nonnegligible fraction of massive $\left[\log \left(M / M_{\odot}\right)>10.5\right]$ bulgeless galaxies which contribute to $\sim 30 \%$ of the total galaxy population number density at $z \gtrsim 0.7$. Overall, we find 2339 out of 19,233 bulgeless galaxies $(12 \%)$ with $\log \left(M / M_{\odot}\right)>10.5$, and $1.5 \%$ of the sample consists of very massive systems with $\log \left(M / M_{\odot}\right)>11$.

The existence of such massive bulgeless galaxies challenges the current picture of galaxy formation, because, in principle, they require a hierarchical growth to reach such large masses. It is not entirely clear how they can increase their masses through mergers without destroying their stellar disks and forming a classical bulge (Toth \& Ostriker 1992). However, it was shown that gas-rich mergers can produce a large disk instead of a spheroidal system (Springel \& Hernquist 2005; Robertson et al. 2006). Disks can survive or rapidly regrow after a merging event provided that the gas fraction in the disks of the progenitors is high (Robertson et al. 2006; Robertson \& Bullock 2008; Hopkins et al. 2009). At $z \sim 1$ massive bulgeless galaxies are less numerous compared to early-type galaxies, but are more abundant than intermediate-type at similar mass range (Figure 8). Their number density decreases with redshift, reducing by a factor of two from $z \sim 1$ to 0.4 , and they are rare in the local universe (Silk \& Mamon 2012). This trend is also found in similar studies of galactic morphological evolution within the same redshift range (Pannella et al. 2009; Oesch et al. 2010). The gradual disappearance of very massive bulgeless galaxies at recent times might imply a process that accompanies the growth of their stellar masses and tends to transform them into an earlier type morphology through the formation of a classical bulge.

To explore this possibility we have used Spitzer and GALEX data to derive both ultraviolet and infrared star forming rates (SFRs), to investigate the star-forming properties of the most massive galaxies in our sample. As mentioned in Section 3.2, Spitzer Space Telescope observations are available for the COSMOS, AEGIS, and GEMS/GOODS-S fields. Overall, 5574 sources $(29 \%)$ out of the entire bulgeless galaxy sample have a good photometric measurement at $24 \mu \mathrm{m}$. Interestingly, $\sim 80 \%$ $(228 / 296)$ of the very massive $\left(M \geqslant 10^{11} M_{\odot}\right)$ bulgeless sample has a $24 \mu \mathrm{m}$ measurement. The total infrared luminosity, $L_{\mathrm{IR}}$ (8-1000 $\mu \mathrm{m})$, is determined following the procedure described in Caputi et al. (2008). Starting from the $24 \mu \mathrm{m}$ flux densities, we used the SED templates of NGC 3351 to reproduce the corresponding rest-frame luminosity and to derive the integrated $L_{\mathrm{IR}}$ of that template. Infrared and ultraviolet SFRs were determined from $L_{\mathrm{IR}}$ and $L_{\mathrm{NUV}}$ rest-frame luminosities, and added together to obtain an estimate of the total SFR for each galaxy.

The SFRs range between 10 and $100 M_{\odot} \mathrm{yr}^{-1}$ and the distribution peaks at roughly $20-30 M_{\odot} \mathrm{yr}^{-1}$ (Figure 10, left panel). For comparison, we plotted the relation found by Noeske et al. (2007) for galaxies with masses $10^{10}-10^{11} M_{\odot}$ at $z=0.2-0.7$ (SF main sequence), and the corresponding line defining a four time higher SFR. Despite the scatter it appears that massive bulgeless have a higher (roughly twice) specific SFR than same mass galaxies located along the SF main sequence, but they do not show evidence of starburst activity which would imply SFR ten time higher than the trend defined by the SF main sequence.

The most massive galaxies in our sample $\left(\log \left(M / M_{\odot}\right)>11\right)$ have an average SFR of about $50 M_{\odot} \mathrm{yr}^{-1}$, implying that they can nearly double their stellar mass due to their star-formation activity over $\sim 2$ Gyr. This is less than the look-back time spanned by our redshift range, corresponding to $\sim 3.5 \mathrm{Gyr}$. These galaxies are clearly different in nature to a fairly massive spiral like the Milky Way. Their SFR is more than 30 times 



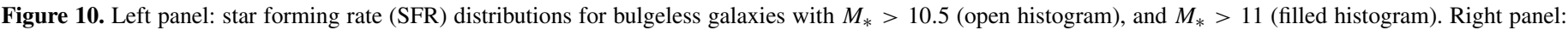

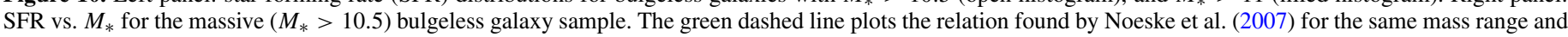
$z=0.2-0.7$. The red dotted line shows the same relation for a four times higher SFR.

(A color version of this figure is available in the online journal.)

higher than the Galactic one, while not reaching the extreme nature of Ultra-luminous Infrared Galaxies. If their intense star-formation activity, as suggested by their high far-infrared luminosities $\left(L_{\mathrm{FIR}} \lesssim 10^{12} L_{\odot}\right)$, is concentrated in the center, it is plausible that a fraction of the additional mass formed from their star-formation activity may lead to the formation of a bulge, contributing to the morphological transformation necessary to evolve into a massive disk galaxy with a bulge. This might explain why massive bulgeless galaxies are relatively rare in the local universe. The presence of a bar may also be related to the morphological transformation of bulgeless galaxies. In the disk-instability scenario, low angular momentum material in the center of a disk is assumed to form a bar due to a global instability (Shen \& Sellwood 2003). Galactic bars induce inflow of interstellar gas towards the center, and the gas accumulated at the galactic center provides raw material for the bulge component (Friedli \& Benz 1993).

Finally, one cannot exclude that the disappearance of massive bulgeless galaxies is related to mergers producing bulgedominated disks or spheroidals. Thus, it is important to analyze the local environment where these systems reside in order to investigate this possibility in more detail. The study of the environmental properties of massive bulgeless galaxies, as well as a more detailed analysis of the multi-color HST images of these systems to search for centrally enhanced star-formation activity or the presence of bars, will be the focus of future papers.

\section{SYSTEMATIC SEARCH OF BULGELESS AGN HOSTS IN X-RAY SURVEYS}

Having assembled a statistically significant sample of bulgeless galaxies at intermediate redshift, we carried out a systematic search for AGN candidates to verify their existence in pure disk systems. X-ray emission is generally recognized as a robust indicator of AGN activity since it does not suffer from heavy dust or gas extinction and contamination from star-formation in the host galaxy is relatively weak.

$\mathrm{X}$-rays from AGNs are produced in the inner and hottest nuclear region of the galaxy, where accretion onto the $\mathrm{BH}$ occurs, and their penetrating power (especially for hard X-rays, $2-10 \mathrm{keV}$ ) allows them to carry information from the central engine without being substantially affected by absorption. Since detection in the soft band (0.5-2 keV) only could miss obscured AGNs (Comastri \& Fiore 2004), we assembled a data set including objects detected in either hard or soft X-ray bands. However, even the deepest of the current X-ray surveys are likely to miss the most heavily obscured "Compton thick" AGNs (Gilli et al. 2007).

\subsection{Identification of $A G N$}

In order to find X-ray emitting bulgeless, we matched the X-ray catalogs described in Section 3.3 with our optical database-including all the morphological types-using an adaptation of the likelihood-ratio method (Sutherland \& Saunders 1992). This method assigns to every matching pair a probability of being a non-false match; then we chose only the pairs for which the method gave high probability values $(P>90 \%)$, and selected only the bulgeless objects in the redshift range of interest $(n \leqslant 1.5$ and $0.4 \leqslant z \leqslant 1.0)$.

We also visually inspected the optical images to spot any obvious mis-identification due to a failure of the matching algorithm (e.g., because of a nearby very bright source). We excluded from our sample extended X-ray sources, which are usually related to galaxy clusters rather than AGNs (Finoguenov et al. 2007), and objects which have large cross-band positional offsets ( $>2.5$ arcsec, Alexander et al. 2003) to obtain a reliable final sample.

The rest-frame X-ray luminosity was calculated using photometric (or spectroscopic, when available) redshifts using the formula

$$
L_{\mathrm{X}}=4 \pi d_{L}^{2} f_{\mathrm{X}}(1+z)^{(-2+\Gamma)},
$$

where $d_{L}$ is the luminosity distance in $\mathrm{cm}$, and $f_{\mathrm{X}}$ is the observed full X-ray flux in units of erg $\mathrm{cm}^{-2} \mathrm{~s}^{-1}$. The photon index, $\Gamma$, is assumed to be equal to 1.8 for all sources, as the use of individual indices results in only minor differences in the restframe luminosities (Barger et al. 2002, 2007).

Following Szokoly et al. (2004) the criteria adopted to identify and classify an AGN are the following:

1. AGN-1: $10^{42} \leqslant L_{\mathrm{X}}<10^{44} \mathrm{erg} \mathrm{s}^{-1}$ and HR $\leqslant-0.2$, which show narrow and broad lines in the optical bands; 
Table 4

X-Ray-selected AGNs

\begin{tabular}{|c|c|c|c|c|c|c|c|}
\hline ID & Morph ID & Survey & $z_{\text {spec }}$ & $z_{\text {phot }}$ & $L_{\mathrm{X}}$ & HR & AGN Type \\
\hline 1 & 13033960 & AEGIS-X & 0.763 & $\ldots$ & $5.352 \times 10^{42}$ & -0.36 & I \\
\hline 2 & 20006036 & XMM-COSMOS & 0.661 & 0.6978 & $5.448 \times 10^{43}$ & 0.47 & II \\
\hline 3 & 20016006 & C-COSMOS & 0.932 & $\ldots$ & $1.181 \times 10^{43}$ & 0.23 & I \\
\hline 4 & 20016854 & C-COSMOS & 0.853 & $\ldots$ & $1.152 \times 10^{43}$ & 1.0 & I \\
\hline 5 & 20038166 & C-COSMOS & 0.654 & $\ldots$ & $2.157 \times 10^{42}$ & -1.0 & II \\
\hline 6 & 20043608 & C-COSMOS & 0.497 & 0.474 & $1.248 \times 10^{42}$ & 1.0 & I \\
\hline 8 & 20091912 & C-COSMOS & 0.984 & $\ldots$ & $7.940 \times 10^{42}$ & -1.0 & II \\
\hline 9 & 20096276 & C-COSMOS & 0.847 & $\ldots$ & $9.176 \times 10^{42}$ & -0.29 & II \\
\hline 10 & 20101857 & C-COSMOS & 0.651 & 0.6328 & $5.340 \times 10^{42}$ & -0.17 & I \\
\hline 11 & 20116906 & C-COSMOS & 0.717 & $\ldots$ & $2.955 \times 10^{42}$ & 1.0 & I \\
\hline 12 & 20124624 & C-COSMOS & 0.934 & $\ldots$ & $6.306 \times 10^{42}$ & 1.0 & I \\
\hline 13 & 20145070 & XMM-COSMOS & 0.804 & $\ldots$ & $5.517 \times 10^{43}$ & -0.52 & I \\
\hline 14 & 20183465 & C-COSMOS & 0.728 & $\ldots$ & $7.158 \times 10^{42}$ & -1.0 & II \\
\hline 16 & 20195289 & XMM-COSMOS & 0.968 & $\ldots$ & $2.762 \times 10^{43}$ & -0.42 & I(II spec) \\
\hline 17 & 50007691 & CDF-N & 0.77 & 0.7517 & $3.416 \times 10^{41}$ & -0.078 & II \\
\hline 18 & 50016371 & CDF-N & 0.56 & 0.6645 & $7.635 \times 10^{41}$ & -0.052 & II \\
\hline 19 & 90002361 & E-CDF-S & 0.673 & $\ldots$ & $5.615 \times 10^{41}$ & 0.162 & II \\
\hline 20 & 90003063 & E-CDF-S & 0.755 & $\ldots$ & $6.739 \times 10^{42}$ & -0.26 & I \\
\hline 21 & 90004178 & E-CDF-S & 0.960 & $\ldots$ & $6.621 \times 10^{42}$ & 0.050 & II \\
\hline 22 & 90004955 & E-CDF-S & 0.633 & $\ldots$ & $1.605 \times 10^{43}$ & -0.049 & II \\
\hline 23 & 90011317 & CDF-S & 0.622 & $\ldots$ & $6.281 \times 10^{42}$ & 0.550 & II \\
\hline 24 & 90011476 & E-CDF-S & 0.572 & $\ldots$ & $2.262 \times 10^{43}$ & -0.059 & II \\
\hline 25 & 90014273 & CDF-S & 0.750 & $\ldots$ & $5.598 \times 10^{42}$ & 0.386 & II \\
\hline 26 & 90021223 & CDF-S & 0.507 & $\ldots$ & $1.943 \times 10^{42}$ & 0.299 & II \\
\hline 27 & 90021708 & CDF-S & 0.628 & $\ldots$ & $2.865 \times 10^{41}$ & 0.089 & II \\
\hline 28 & 90028848 & E-CDF-S & 0.686 & 0.72 & $8.066 \times 10^{42}$ & 0.039 & II \\
\hline 29 & 90033648 & CDF-S & 0.789 & 0.784 & $2.821 \times 10^{42}$ & -0.104 & II \\
\hline 30 & 90038381 & E-CDF-S & 0.554 & $\ldots$ & $2.090 \times 10^{42}$ & 0.423 & II \\
\hline
\end{tabular}

Notes. Column (1) indicates the catalog object ID. Column (2) lists the original X-ray catalog of each source. Columns (3) and (4) refer to the spectroscopic and photometric redshift. Column (5) is the rest-frame luminosity expressed in erg s${ }^{-1}$. Column (6) is the hardness ratio. Column (7) indicates the AGN type.

2. AGN-2: $10^{41} \leqslant L_{\mathrm{X}}<10^{44} \mathrm{erg} \mathrm{s}^{-1}$ and HR $\geqslant-0.2$, for which only narrow lines are observed in the optical bands. where HR is the hardness ratio defined as $H-S / H+S ; H$ is the number of photon counts in the hard band and $S$ is the number of counts in the soft band. The HR can be used to roughly estimate the obscuration affecting the sources, as the most efficient and reddening independent method to select obscured type 2 AGNs is the presence of luminous X-ray emission and hard X-ray colors. Where a source has only upper limits in the soft or hard bands, the HR is assumed as +1 and -1 , respectively.

Though widely used, this method suffers from some uncertainties. It is worth noting that some high redshift absorbed/ type 2 sources can be identified as type 1 when adopting a X-ray classification based on the HR. In fact, increasing absorption makes the sources harder, while a high redshift makes them softer. Nevertheless, there is evidence that the method is robust as it gives results that are in good agreement with those obtained through optical indicators, such as Baldwin, Phillips, \& Terlevich (BPT) diagrams (see for instance, El Bouchefry 2009). This classification yielded 43 AGN candidates.

Typically, hard X-ray detections with luminosity higher that $10^{41} \mathrm{erg} \mathrm{s}^{-1}$ are evident tracers of AGN activity; nonetheless, for sources in which only soft X-rays were detected (21 in our sample), we tested whether the X-ray luminosities could be produced by star-formation activity instead of an AGN. SFRs derived in Section 5.3 for most of the AGN candidates give values in the range $4-50 M_{\odot} \mathrm{yr}^{-1}$, with just one outlier showing
$167 M_{\odot} \mathrm{yr}^{-1}$. Applying the relation (Mineo et al. 2014)

$$
L_{\mathrm{X}} / \mathrm{SFR}=(3.5 \pm 0.4) \times 10^{39}\left(\mathrm{erg} \mathrm{s}^{-1} / M_{\odot} \mathrm{yr}^{-1}\right),
$$

which holds at $0.1 \leqslant z \leqslant 1.3$, we can conclude that the $\mathrm{X}$-ray luminosities of our sample are one to three orders of magnitude higher than the ones calculated with Equation (3).

\subsection{Morphological Analysis of AGN Candidates}

The morphological classification of the sample extracted from the ACS-GC, as described in Griffith et al. (2012) and in Section 2.1, relied on GALFIT results; the reliability of such a classification was assessed in Section 4.1, and it proved to be appropriate for statistical purposes. However, since the AGN host candidates represent a very small fraction of our sample, we double checked the morphology of each object with an X-ray counterpart to exclude possible mis-classifications due to the automatic procedure. Given that bulgeless AGNs are extremely rare, any morphological mis-classification would likely be clearly revealed in this sample.

For each target we retrieved a $256 \times 256$ pixel cutout from the HST/ACS archive. We visually inspected the 43 cutout images and identified four sources which we classified as mergers and rejected from the sample without attempting any further analysis. One additional source showed an extended dust lane feature and was also discarded. The morphology of the remaining sources was tested with a detailed analysis of 
Table 5

Morphological Parameters from Surface Brightness Profile Fitting Using a Single Sérsic Profile or a Combination of a Sérsic Profile Plus an Exponential Disk

\begin{tabular}{|c|c|c|c|c|c|c|}
\hline ID & ID MORPH & $\begin{array}{c}\mu_{e} \\
\left(\operatorname{mag~arcsec}^{-2}\right)\end{array}$ & $\begin{array}{c}R_{e} \\
(\operatorname{arcsec})\end{array}$ & $n$ & $\begin{array}{c}\mu_{d}^{0} \\
\left(\mathrm{mag} \mathrm{arcsec}^{-2}\right)\end{array}$ & $\begin{array}{c}h_{d} \\
(\operatorname{arcsec})\end{array}$ \\
\hline 1 & 13033960 & $21.972 \pm 0.023$ & $0.250 \pm 0.004$ & $0.87 \pm 0.02$ & $21.530 \pm 0.048$ & $0.89 \pm 0.03$ \\
\hline 2 & 20006036 & $22.831 \pm 0.038$ & $0.538 \pm 0.020$ & $0.44 \pm 0.04$ & $\ldots$ & $\ldots$ \\
\hline 3 & 20016006 & $22.166 \pm 0.005$ & $0.416 \pm 0.003$ & $0.80 \pm 0.01$ & $\ldots$ & $\ldots$ \\
\hline 4 & 20016854 & $20.469 \pm 0.029$ & $0.106 \pm 0.002$ & $0.62 \pm 0.02$ & $21.004 \pm 0.028$ & $0.31 \pm 0.01$ \\
\hline 5 & 20038166 & $24.051 \pm 0.021$ & $0.371 \pm 0.005$ & $1.16 \pm 0.03$ & $\ldots$ & $\ldots$ \\
\hline 6 & 20043608 & $23.278 \pm 0.024$ & $1.168 \pm 0.013$ & $1.25 \pm 0.03$ & $\ldots$ & $\ldots$ \\
\hline 7 & 20067818 & $21.464 \pm 0.007$ & $0.206 \pm 0.001$ & $0.78 \pm 0.01$ & $\ldots$ & $\ldots$ \\
\hline 8 & 20091912 & $23.834 \pm 0.005$ & $0.923 \pm 0.006$ & $0.93 \pm 0.01$ & $\ldots$ & $\ldots$ \\
\hline 9 & 20096276 & $21.776 \pm 0.013$ & $0.190 \pm 0.002$ & $0.82 \pm 0.01$ & $\ldots$ & $\ldots$ \\
\hline 10 & 20101857 & $21.466 \pm 0.055$ & $0.112 \pm 0.004$ & $0.71 \pm 0.05$ & $20.647 \pm 0.010$ & $0.68 \pm 0.01$ \\
\hline 11 & 20116906 & $21.277 \pm 0.009$ & $0.280 \pm 0.002$ & $0.73 \pm 0.01$ & $\ldots$ & $\ldots$ \\
\hline 12 & 20124624 & $23.386 \pm 0.017$ & $0.664 \pm 0.008$ & $0.92 \pm 0.01$ & $\ldots$ & $\ldots$ \\
\hline 13 & 20145070 & $23.733 \pm 0.038$ & $0.139 \pm 0.003$ & $0.55 \pm 0.03$ & $22.080 \pm 0.013$ & $0.92 \pm 0.01$ \\
\hline 14 & 20183465 & $21.737 \pm 0.011$ & $0.447 \pm 0.004$ & $0.85 \pm 0.01$ & $\ldots$ & $\ldots$ \\
\hline 15 & 20188370 & $24.525 \pm 0.009$ & $0.612 \pm 0.007$ & $0.95 \pm 0.02$ & $\ldots$ & $\ldots$ \\
\hline 16 & 20195289 & $23.153 \pm 0.023$ & $0.474 \pm 0.011$ & $0.55 \pm 0.02$ & $\ldots$ & $\ldots$ \\
\hline 17 & 50007691 & $21.653 \pm 0.022$ & $0.197 \pm 0.002$ & $0.57 \pm 0.01$ & $20.945 \pm 0.023$ & $0.34 \pm 0.01$ \\
\hline 18 & 50016371 & $20.086 \pm 0.013$ & $0.099 \pm 0.001$ & $0.61 \pm 0.01$ & $20.180 \pm 0.005$ & $0.32 \pm 0.01$ \\
\hline 19 & 90002361 & $21.995 \pm 0.159$ & $0.178 \pm 0.022$ & $0.33 \pm 0.07$ & $21.077 \pm 0.024$ & $0.78 \pm 0.01$ \\
\hline 20 & 90003063 & $21.541 \pm 0.011$ & $0.426 \pm 0.005$ & $0.55 \pm 0.02$ & $\ldots$ & $\ldots$ \\
\hline 21 & 90004178 & $23.584 \pm 0.086$ & $0.534 \pm 0.030$ & $0.84 \pm 0.11$ & $\ldots$ & $\ldots$ \\
\hline 22 & 90004955 & $22.945 \pm 0.047$ & $0.319 \pm 0.009$ & $1.06 \pm 0.05$ & $\ldots$ & $\ldots$ \\
\hline 23 & 90011317 & $19.546 \pm 0.003$ & $0.246 \pm 0.001$ & $0.77 \pm 0.01$ & $\ldots$ & $\ldots$ \\
\hline 24 & 90011476 & $19.900 \pm 0.004$ & $0.245 \pm 0.001$ & $1.19 \pm 0.01$ & $\ldots$ & $\ldots$ \\
\hline 25 & 90014273 & $20.428 \pm 0.014$ & $0.467 \pm 0.006$ & $0.94 \pm 0.01$ & $\ldots$ & $\ldots$ \\
\hline 26 & 90021223 & $21.382 \pm 0.601$ & $0.149 \pm 0.044$ & $1.07 \pm 0.18$ & $\ldots$ & $\ldots$ \\
\hline 27 & 90021708 & $21.235 \pm 0.009$ & $0.202 \pm 0.002$ & $0.84 \pm 0.01$ & $\ldots$ & $\ldots$ \\
\hline 28 & 90028848 & $23.103 \pm 0.110$ & $0.519 \pm 0.038$ & $0.96 \pm 0.11$ & $\ldots$ & $\ldots$ \\
\hline 29 & 90033648 & $21.371 \pm 0.402$ & $0.065 \pm 0.018$ & $0.53 \pm 0.18$ & $20.546 \pm 0.015$ & $0.43 \pm 0.01$ \\
\hline 30 & 90038381 & $21.211 \pm 0.008$ & $0.374 \pm 0.002$ & $1.37 \pm 0.01$ & $\ldots$ & $\ldots$ \\
\hline
\end{tabular}

Notes. Column (1) indicates the catalog object ID. Columns (2)-(4) refer to the Sérsic profile parameters: surface brightness, $\mu_{e}$, scale length, $R_{e}$, and the Sérsic index $n$. Columns (5) and (6) are the corresponding disk profile coefficients.

the surface brightness (SB) profiles. We measured the surface photometry in IRAF, using the task ellipse in STSDAS. We then determined the best fit Sérsic profiles for each AGN host candidate galaxy. If a single Sérsic model did not provide an adequate fit to the data, we tried a combination of a Sérsic plus an exponential disk profile. According to Kormendy \& Kennicutt (2004) late-type galaxies can host pseudo-bulges in the central regions which are described by low Sérsic indices $(n<2)$, and have central light distribution similar to that of the outer disk. From this analysis we rejected three candidates showing a bulge component with $n>1.5$. Finally, five additional objects were rejected because they had an ambiguous match between the optical and the X-ray source position.

The final sample is formed by thirty objects, eleven of them are classified as type I, the remaining nineteen are type II AGN. Their X-ray properties are displayed in Table 4 . Their brightness surface profiles are described by either a single Sérsic profile with $n<1.5$ (22 out of 30;74\%), or a combination of a central "pseudo-bulge" Sérsic profile with $n<1.5$ plus an extended disk ( 8 out of $30 ; 26 \%)$. The best-fit parameters are collected in Table 5. Figures 11 and 12 show the HST images of the 30 AGN host galaxy candidates and the SB profiles with the corresponding best fits. Substructures in the disks are visible in some cases affecting the goodness of the fit.

Visual inspection of the images shows that among the sample there are six targets which, despite the low $n$ index, show a compact/spheroidal morphology (Figure 12). Color-mass and color-color plots of these galaxies show that they are at the edge of the red sequence (red dots, Figure 13, left panel). However, only two objects fall in the $U V J$ region of red quiescent galaxies. The remaining ones appear to be star-forming systems with a substantial amount of dust (red dots, Figure 13, right panel). Although these galaxies should be treated with additional care, we show that they do have low Sérsic indices and most of them have evidence of star-formation activity, thus suggesting that they can be considered as reliable bulgeless AGN host candidates.

A more detailed analysis of the X-ray properties of the AGN candidates, including an estimate of the $\mathrm{BH}$ masses, will be presented in a forthcoming paper (E. Leonardo et al. 2013, in preparation).

\section{SUMMARY AND CONCLUSION}

In this paper we presented a magnitude-limited $\left(m_{\mathrm{AB}}<24\right)$, low-inclination, point-source free, $K$-corrected sample of 19,233 bulgeless galaxies at intermediate redshift $(0.4 \leqslant z \leqslant 1)$ based on the ACS-GC public morphology catalog. It includes a photometric and morphological database derived from the COSMOS, AEGIS, GEMS, GOODS-N, and GOODS-S surveys. We assembled all the ancillary data available for these five fields and generated a homogeneous data set, including photometric measurements from the far-ultraviolet to midinfrared wavelength range. The catalog is made publicly available to the scientific community and it contains morphological 



Figure 11. HST/ACS images and surface brightness profile of the AGN bulgeless host galaxy candidates with $n<1.5$ and disk/irregular morphology. (A color version of this figure is available in the online journal.)

classifications, photometric and/or spectroscopic redshifts, restframe magnitudes, and stellar masses which have been estimated by fitting the multi-color photometry to a grid of composite stellar population models using the kcorrect code. Comparison with existing catalogs of COSMOS and GOODS-S fields (Pannella et al. 2009; Marchesini et al. 2009) shows that our results are in agreement with both the morphological classification and mass estimates already available in the literature. The applied magnitude limit in the $I$ band corresponds to a minimum detectable mass of $10^{9} M_{\odot}$ and $4.3 \times 10^{10} M_{\odot}$ at $z=0.4$ and $z=$ 1 , respectively.

We analyzed the properties of the sample and the evolution of pure-disk systems with redshift. As expected, the bulk of bulgeless galaxies occupies the low-mass end region of the color-mass diagram $\left(\log \left(M_{\star} / M_{\odot}\right)<10\right)$ and is predominantly blue, indicating recent star-formation activity, as opposed to the early-type objects, which dominate the red, high-mass end of the diagram.

Within the bulgeless sample, we found a non-negligible population of very massive galaxies with $\log \left(M_{\star} / M_{\odot}\right)>10.5$, which contributes to $\sim 30 \%$ of the total galaxy population number density at $z \gtrsim 0.7$. Analysis of the evolution of the number density of the different morphological types with redshift, above our completeness mass limit $\left(3 \times 10^{10} M_{\odot}\right)$, shows a decrease of the bulgeless number density with time compared to the "bulgy"/early-type systems. This implies that 

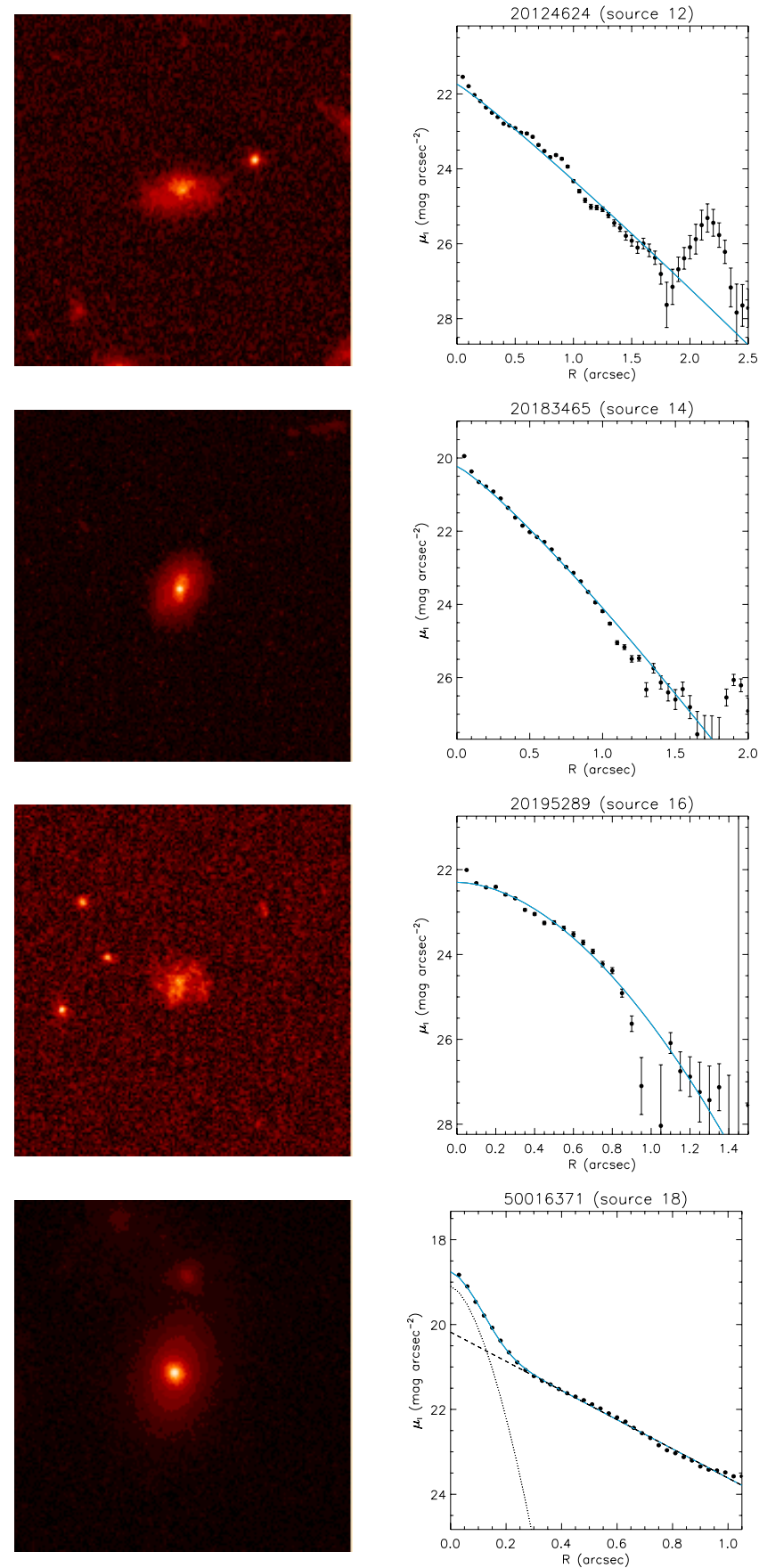
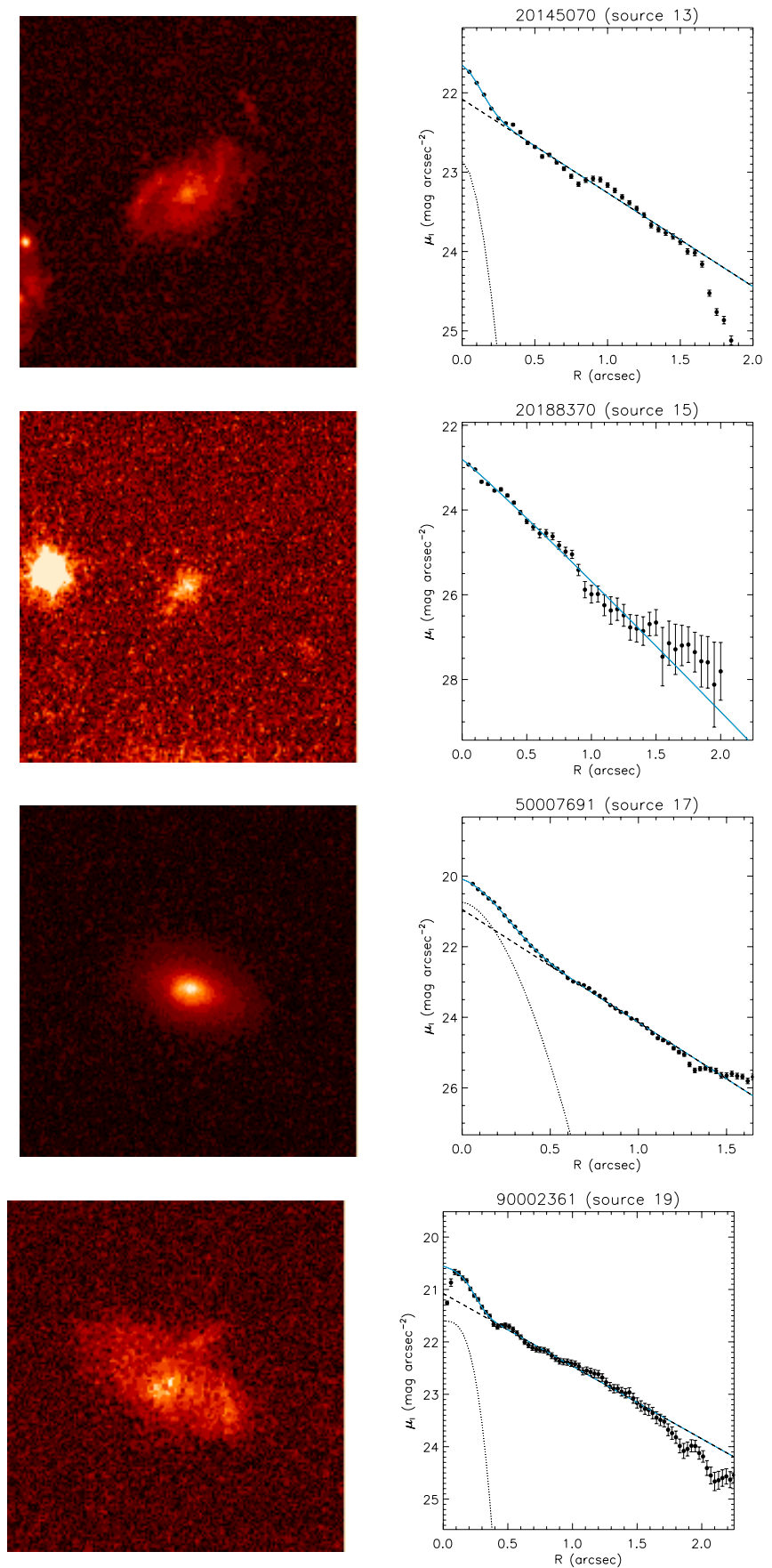

Figure 11. (Continued)

internal bulge growth through either star-formation activity or mergers, and interactions with nearby companions make massive pure-disk systems evolve to earlier type morphologies through time.

The most massive galaxies among our sample are indeed characterized by SFRs ranging between 10 and $100 M_{\odot} \mathrm{yr}^{-1}$, suggesting that a prolonged star-formation activity $\left(>10^{8} \mathrm{yr}\right)$ at such rate might contribute to the build-up of nuclear mass and formation of a classical bulge. The disappearance of very massive bulgeless galaxies is confirmed by low redshift studies. Pure disk/pseudo-bulge galaxies are the most common morphological types within the local volume $(<11 \mathrm{Mpc})$ at stellar masses below $10^{10} M_{\odot}$, while more massive pure-disk galaxies are rare (Fisher \& Drory 2010).

Since bulges and AGN are thought to co-evolve in galaxies, we searched for possible AGN counterparts to bulgeless systems, in order to investigate the formation of massive nuclear BHs in galaxies with an apparently uneventful merger history. Because X-ray emission provides one of the most effective and reliable tools to confirm the existence of an AGN compared to the radio continuum or mid-infrared observations, we combined the optical database with X-ray catalogs to search for X-ray sources within our sample. We found only 30 X-ray detections with a reliable bulgeless optical counterpart, after 

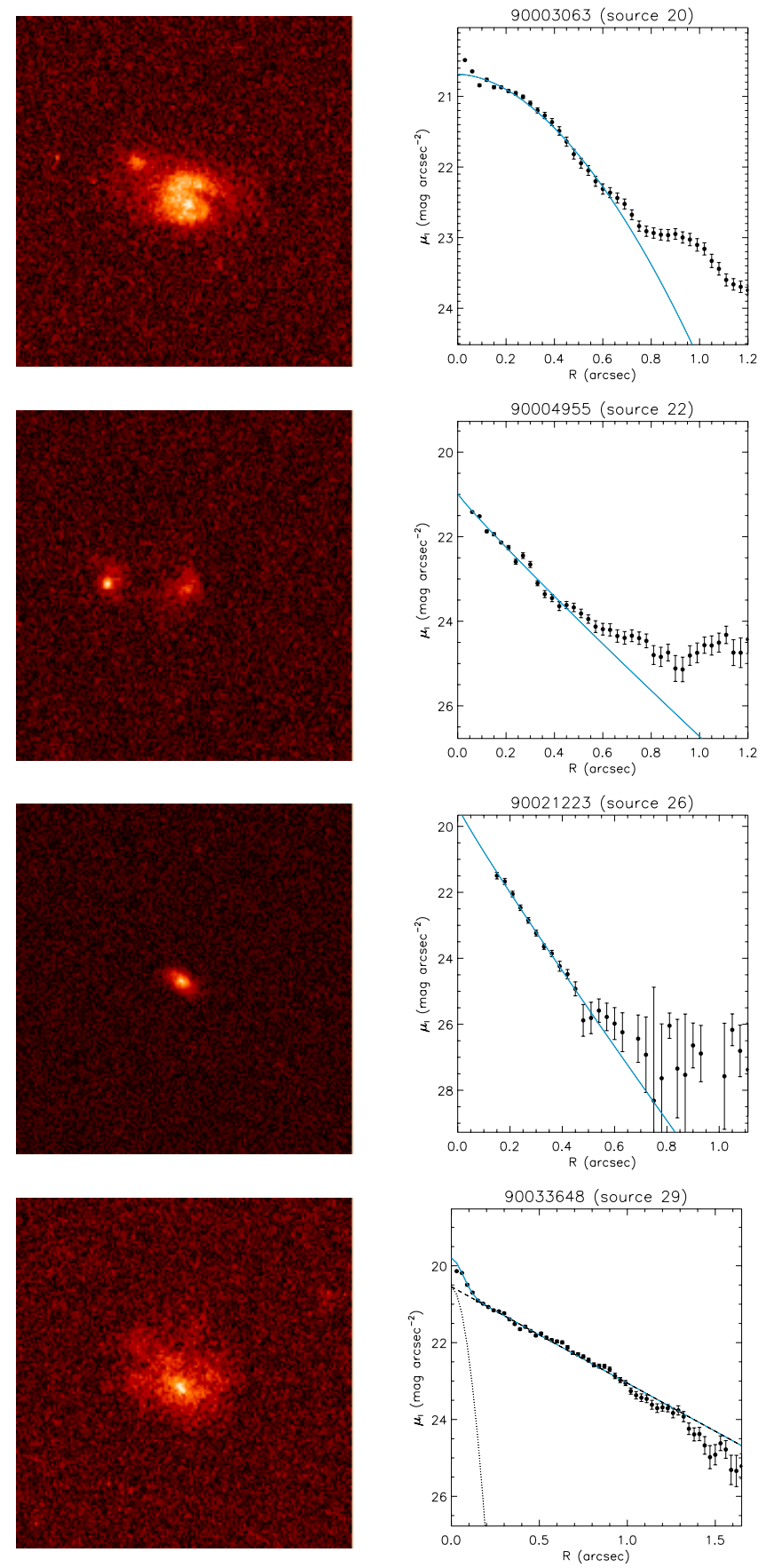
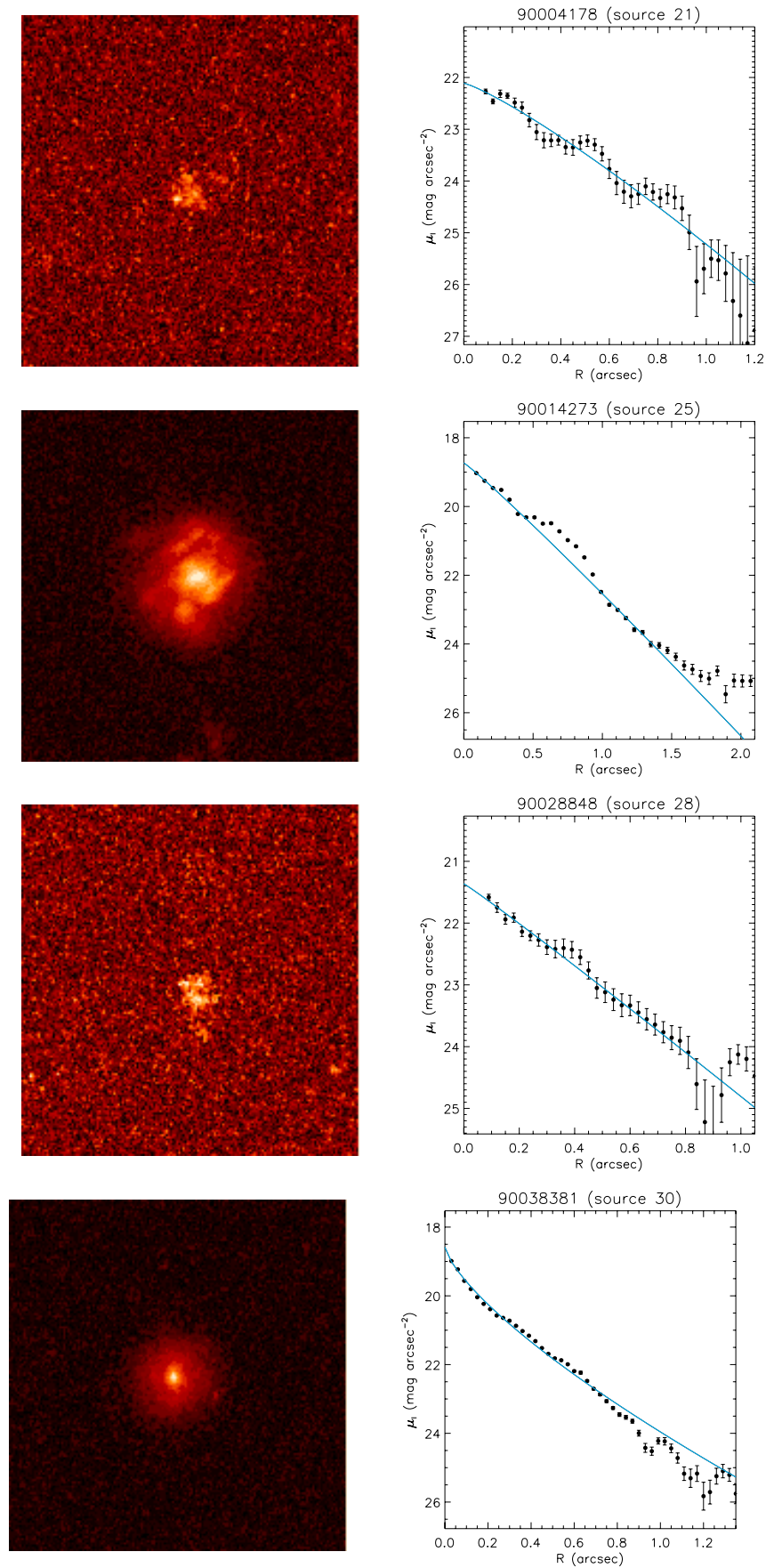

Figure 11. (Continued)

having visually inspected the corresponding HST images and re-analyzed the $I$-band surface brightness profiles. These AGN host candidates are well-fit by either a single Sérsic model with index $n<1.5$ or by a combination of a pseudo-bulge (with $n<1.5$ ) and a disk component.

The 30 detections represent $0.2 \%$ of the bulgeless sample; this fraction must be considered as a stringent lower limit. Comparing with the other morphological classes, we found that this detection ratio is only marginally smaller than the one of the intermediate-type population $(0.3 \%)$ and six times smaller than that of the bulge-dominated sample (1.2\%).

We thank the anonymous referee for the useful comments and suggestions. This work is based on (GO-10134, GO-09822,
GO-09425.01, GO-09583.01, GO-9500) program observations with the NASA/ESA Hubble Space Telescope, obtained at the Space Telescope Science Institute, which is operated by the Association of Universities for Research in Astronomy, Inc., under NASA contract NAS 5-26555. This research has made use of the NASA/IPAC Infrared Science Archive, which is operated by the Jet Propulsion Laboratory, California Institute of Technology, under contract with the National Aeronautics and Space Administration. This work is also based on zCOSMOS observations carried out using the Very Large Telescope at the ESO Paranal Observatory under Program ID: LP175.A-0839. This study makes use of data from AEGIS, a multiwavelength sky survey conducted with the Chandra, GALEX, Hubble, Keck, CFHT, MMT, Subaru, Palomar, Spitzer, 

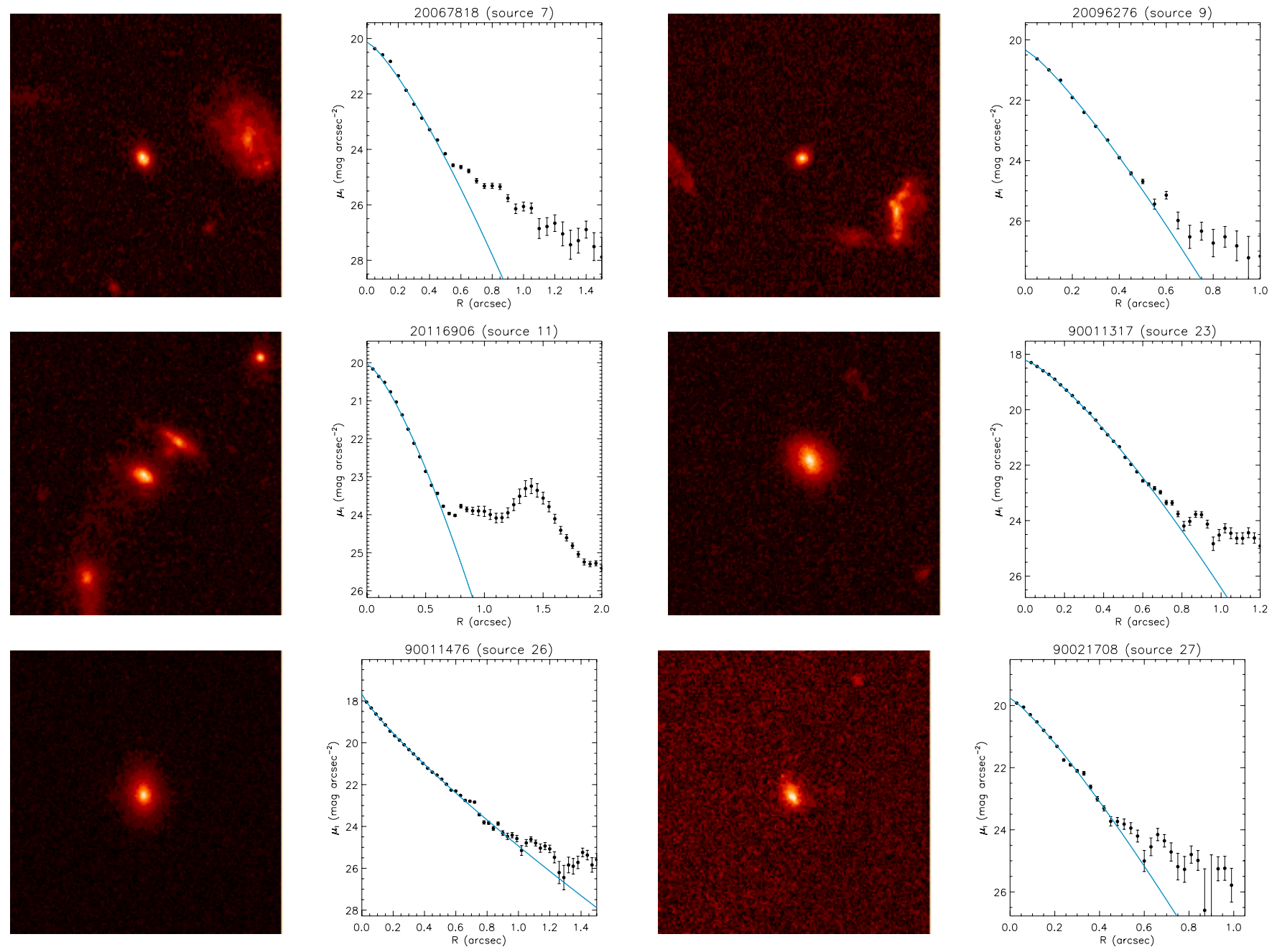

Figure 12. HST/ACS images and surface brightness profile of the AGN bulgeless host galaxy candidates with $n<1.5$ and spheroidal morphology.

(A color version of this figure is available in the online journal.)


Figure 13. Left panel: $g-r$ color-mass plot of the AGN bulgeless host galaxy candidates with disk/irregular (blue triangles) and spheroidal (red dots) morphology $(0.4 \leqslant z \leqslant 1.0)$. Right panel: rest-frame $U-V$ as a function of $V-J$ color for the same galaxy sample. Superimposed as dashed lines are the rest-frame colors cuts defined in Williams et al. (2009).

(A color version of this figure is available in the online journal.) 
VLA, and other telescopes and supported in part by the NSF, NASA, and the STFC. Funding for the DEEP2 survey has been provided by NSF grants AST95-09298, AST-0071048, AST-0071198, AST-0507428, and AST-0507483 as well as NASA LTSA grant NNG04GC89G. Some of the data were obtained at the W. M. Keck Observatory, which is operated as a scientific partnership among the California Institute of Technology, the University of California, and the National Aeronautics and Space Administration. The Observatory was made possible by the generous financial support of the W. M. Keck Foundation. Work on this paper is based on observations obtained with MegaPrime/MegaCam, a joint project of Canada-FranceHawaii Telescope (CFHT) and CEA/DAPNIA, at the CFHT, which is operated by the National Research Council (NRC) of Canada, the Institut National des Science de l'Univers of the Centre National de la Recherche Scientifique (CNRS) of France, and the University of Hawaii. We gratefully acknowledge the GOODS Team (http://cosmos.astro.caltech.edu) for providing all the imaging material available worldwide. We are also indebted to the COMBO-17 (Wolf et al. 2004) and MUSIC teams (Grazian et al. 2006) for the catalogs publicly supplied. The authors gratefully acknowledge financial support from the Science and Technology Foundation (FCT, Portugal) through research grants PTDC/CTE-AST/105287/2008, PTDC/FISAST/2194/2012, PEst-OE/FIS/UI2751/2011, and PEst-OE/ FIS/UI2751/2014. L.B., E.L., and M.E.F. gratefully acknowledge support from the Science and Technology Foundation (FCT, Portugal) through fellowships SFRH/BPD/62966/2009, SFRH/BPD/71278/2010, and SFRH/BPD/36141/2007.

\section{REFERENCES}

Alexander, D. M., Bauer, F. E., Brandt, W. N., et al. 2003, AJ, 126, 539 Araya Salvo, C., Mathur, S., Ghosh, H., Fiore, F., \& Ferrarese, L. 2012, ApJ, 757,179

Barden, M., Häußler, B., Peng, C. Y., McIntosh, D. H., \& Guo, Y. 2012, MNRAS, 422, 449

Barger, A. J., Cowie, L. L., Brandt, W. N., et al. 2002, AJ, 124, 1839

Barger, A. J., Cowie, L. L., \& Wang, W.-H. 2007, ApJ, 654, 764

Barger, A. J., Cowie, L. L., \& Wang, W.-H. 2008, ApJ, 689, 687

Barmby, P., Huang, J.-S., Ashby, M. L. N., et al. 2008, ApJS, 177, 431

Barro, G., Pérez-González, P. G., Gallego, J., et al. 2011, ApJS, 193, 13

Barth, A. J., Strigari, L. E., Bentz, M. C., Greene, J. E., \& Ho, L. C. 2009, ApJ, 690, 1031

Bell, E. F. 2008, ApJ, 682, 355

Bell, E. F., \& de Jong, R. S. 2001, ApJ, 550, 212

Bell, E. F., van der Wel, A., Papovich, C., et al. 2012, ApJ, 753, 167

Bertin, E., \& Arnouts, S. 1996, A\&AS, 117, 393

Blanton, M. R., \& Roweis, S. 2007, AJ, 133, 734

Brook, C. B., Stinson, G., Gibson, B. K., et al. 2012, MNRAS, 419, 771

Brusa, M., Civano, F., Comastri, A., et al. 2010, ApJ, 716, 348

Bruzual, G., \& Charlot, S. 2003, MNRAS, 344, 1000

Bundy, K., Ellis, R. S., Conselice, C. J., et al. 2006, ApJ, 651, 120

Bundy, K., Fukugita, M., Ellis, R. S., et al. 2009, ApJ, 697, 1369

Bundy, K., Scarlata, C., Carollo, C. M., et al. 2010, ApJ, 719, 1969

Cairós, L. M., Vílchez, J. M., González Pérez, J. N., Iglesias-Páramo, J., \& Caon, N. 2001, ApJS, 133, 321

Caldwell, J. A. R., McIntosh, D. H., Rix, H.-W., et al. 2008, ApJS, 174, 136

Capak, P., Aussel, H., Ajiki, M., et al. 2007, ApJS, 172, 99

Cappelluti, N., Brusa, M., Hasinger, G., et al. 2009, A\&A, 497, 635

Caputi, K. I., Lilly, S. J., Aussel, H., et al. 2008, ApJ, 680, 939

Cassata, P., Cimatti, A., Franceschini, A., et al. 2005, MNRAS, 357, 903

Cassata, P., Guzzo, L., Franceschini, A., et al. 2007, ApJS, 172, 270

Chambers, K. C., Miley, G. K., van Breugel, W. J. M., \& Huang, J.-S. 2004, MNRAS, 351, 1215

Coelho, B., Antón, S., Lobo, C., \& Ribeiro, B. 2013, MNRAS, 436, 2426

Coil, A. L., Newman, J. A., Kaiser, N., et al. 2004, ApJ, 617, 765

Cole, S., Lacey, C. G., Baugh, C. M., \& Frenk, C. S. 2000, MNRAS, 319, 168

Collins, C. A., Stott, J. P., Hilton, M., et al. 2009, Natur, 458, 603

Comastri, A., \& Fiore, F. 2004, ApJS, 294, 63
Cowie, L. L., Barger, A. J., Hu, E. M., Capak, P., \& Songaila, A. 2004, AJ 127,3137

Daddi, E., Cimatti, A., Renzini, A., et al. 2004, ApJ, 617, 746

Dahlen, T., Mobasher, B., Dickinson, M., et al. 2010, ApJ, 724, 425

Davis, M., Faber, S. M., Newman, J., et al. 2003, Proc. SPIE, 4834, 161

Davis, M., Guhathakurta, P., Konidaris, N. P., et al. 2007, ApJL, 660, L1

Desroches, L. B., \& Ho, L. C. 2009, ApJ, 690, 267

Dickinson, M., Giavalisco, M., \& GOODS Team 2003, in The Mass of Galaxies at Low and High Redshift, ed. R. Bender \& A. Renzini (Berlin: Springer), 324

D’Onghia, E., \& Burkert, A. 2004, ApJL, 612, L13

Drory, N., Bender, R., \& Hopp, U. 2004, ApJL, 616, L103

Dutton, A. A., \& van den Bosch, F. C. 2009, MNRAS, 396, 141

El Bouchefry, K. 2009, MNRAS, 396, 2011

Elvis, M., Civano, F., Vignali, C., et al. 2009, ApJS, 184, 158

Fall, S. M., \& Efstathiou, G. 1980, MNRAS, 193, 189

Filippenko, A. V., \& Ho, L. C. 2003, ApJL, 588, L13

Finoguenov, A., Guzzo, L., Hasinger, G., et al. 2007, ApJS, 172, 182

Fisher, D. B., \& Drory, N. 2010, ApJ, 716, 942

Frayer, D. T., Sanders, D. B., Surace, J. A., et al. 2009, AJ, 138, 1261

Friedli, D., \& Benz, W. 1993, A\&A, 268, 65

Gadotti, D. A. 2009, MNRAS, 393, 1531

Giavalisco, M., Ferguson, H. C., Koekemoer, A. M., et al. 2004, ApJL, 600, L93

Gilli, R., Comastri, A., \& Hasinger, G. 2007, A\&A, 463, 79

Grazian, A., Fontana, A., de Santis, C., et al. 2006, A\&A, 449, 951

Griffith, R. L., Cooper, M. C., Newman, J. A., et al. 2012, ApJS, 200, 9

Gwyn, S. D. J. 2008, PASP, 120, 212

Hainline, L. J., Blain, A. W., Smail, I., et al. 2011, ApJ, 740, 96

Häring, N., \& Rix, H. W. 2004, ApJL, 604, L89

Hoaglin, D. C., Mosteller, F., \& Tukey, J. W. 1983, Understanding Robust and Exploratory Data Analysis (New York: Wiley)

Hopkins, P. F., Cox, T. J., Younger, J. D., \& Hernquist, L. 2009, ApJ, 691, 1168

Ilbert, O., Arnouts, S., McCracken, H. J., et al. 2006, A\&A, 457, 841

Ilbert, O., Capak, P., Salvato, M., et al. 2009, ApJ, 690, 1236

Kajisawa, M., Ichikawa, T., Tanaka, I., et al. 2011, PASJ, 63, 379

Kautsch, S. J., Grebel, E. K., Barazza, F. D., \& Gallagher, J. S., III. 2006, A\&A, 445,765

Kewley, L. J., Dopita, M. A., Sutherland, R. S., Heisler, C. A., \& Trevena, J. 2001, ApJ, 556, 121

Koda, J., Milosavljević, M., \& Shapiro, P. R. 2009, ApJ, 696, 254

Kormendy, J., Bender, R., \& Cornell, M. E. 2011, Natur, 469, 374

Kormendy, J., \& Kennicutt, R. C., Jr. 2004, ARA\&A, 42, 603

Kormendy, J., \& Richstone, D. 1995, ARA\&A, 33, 581

Kunth, D., Maurogordato, S., \& Vigroux, L. 1988, A\&A, 204, 10

Laird, E. S., Nandra, K., Georgakakis, A., et al. 2009, ApJ, 180, 102

Larson, D., Dunkley, J., Hinshaw, G., et al. 2011, ApJS, 192, 16

Lehmer, B. D., Brandt, W. N., Alexander, D. M., et al. 2005, ApJS, 161, 21

Lilly, S. J., Le Fèvre, O., Renzini, A., et al. 2007, ApJS, 172, 70

Loose, H.-H., \& Thuan, F. X. 1986a, MitAG, 65, 231

Loose, H.-H., \& Thuan, T. X. 1986b, ApJ, 309, 59

Lotz, J. M., Davis, M., Faber, S. M., et al. 2008, ApJ, 672, 177

Lotz, J. M., Primack, J., \& Madau, P. 2004, AJ, 128, 163

Magorrian, J., Tremaine, S., Richstone, D., et al. 1998, AJ, 115, 2285

Marchesini, D., van Dokkum, P. G., Förster Schreiber, N. M., et al. 2009, ApJ, 701,1765

Mathur, S., Fields, D., Peterson, B. M., \& Grupe, D. 2012, ApJ, 754, 146

McAlpine, W., Satyapal, S., Gliozzi, M., et al. 2011, ApJ, 728, 25

McCracken, H. J., Milvang-Jensen, B., Dunlop, J., et al. 2012, A\&A, 544, A156

McGrath, E. J., Stockton, A., Canalizo, G., Iye, M., \& Maihara, T. 2008, ApJ, 682,303

Mineo, S., Gilfanov, M., Lehmer, B. D., Morrison, G. E., \& Sunyaev, R. 2014, MNRAS, 437, 1698

Moster, B. P., Somerville, R. S., Newman, J. A., \& Rix, H.-W. 2011, ApJ, 731,113

Muzzin, A., Marchesini, D., van Dokkum, P. G., et al. 2009, ApJ, 701, 1839

Noeske, K. G., Weiner, B. J., Faber, S. M., et al. 2007, ApJL, 660, L43

Oesch, P. A., Carollo, C. M., Feldmann, R., et al. 2010, ApJL, 714, L47

Pannella, M., Gabasch, A., Goranova, Y., et al. 2009, ApJ, 701, 787

Peng, C. Y., Ho, L. C., Impey, C. D., \& Rix, H.-W. 2002, AJ, 124, 266

Peterson, B. M., Bentz, M. C., Desroches, L.-B., et al. 2005, ApJ, 632, 799

Reines, A. E., Sivakoff, G. R., Johnson, K. E., \& Brogan, C. L. 2011, Natur, 470, 6

Robertson, B., Bullock, J. S., Cox, T. J., et al. 2006, ApJ, 645, 986

Robertson, B., Yoshida, N., Springel, V., \& Hernquist, L. 2004, ApJ, 606, 32

Robertson, B. E., \& Bullock, J. S. 2008, ApJL, 685, L27

Sanders, D. B., Salvato, M., Aussel, H., et al. 2007, ApJS, 172, 86 
Satyapal, S., Böker, T., Mcalpine, W., et al. 2009, ApJ, 704, 439

Satyapal, S., Vega, D., Heckman, T., O'Halloran, B., \& Dudik, R. 2007, ApJL, 663, L9

Scarlata, C., Carollo, C. M., Lilly, S., et al. 2007, ApJS, 172, 406

Schawinski, K., Urry, C. M., Virani, S., et al. 2010, ApJ, 711, 284

Scoville, N., Abraham, R. G., Aussel, H., et al. 2007, ApJS, 172, 38

Secrest, N. J., Satyapal, S., Gliozzi, M., et al. 2012, ApJ, 753, 38

Sérsic, J. L. 1968, Atlas de galaxias australes (Cordoba, Argentina: Observatorio Astronomico)

Shen, J., \& Sellwood, J. A. 2003, BAAS, 35, 1353

Silk, J., \& Mamon, G. A. 2012, RAA, 12, 917

Simmons, B. D., Lintott, C., Schawinski, K., et al. 2012, MNRAS, 429, 2199

Springel, V., \& Hernquist, L. 2005, ApJL, 622, L9

Suh, H., Jeong, H., Oh, K., et al. 2010, ApJS, 187, 374

Sutherland, W., \& Saunders, W. 1992, MNRAS, 259, 413

Szokoly, G. P., Bergeron, J., Hasinger, G., et al. 2004, ApJS, 155, 271

Taniguchi, Y., Scoville, N., Murayama, T., et al. 2007, ApJS, 172, 9
Thomas, D., Maraston, C., Schawinski, K., Sarzi, M., \& Silk, J. 2010, MNRAS, 404, 1775

Thornton, C. E., Barth, A. J., Ho, L. C., Rutledge, R. E., \& Greene, J. E. 2008, ApJ, 686, 892

Toth, G., \& Ostriker, J. P. 1992, ApJ, 389, 5

Tremaine, S., Gebhardt, K., Bender, R., et al. 2002, ApJ, 574, 740

van der Wel, A., Rix, H.-W., Wuyts, S., et al. 2011, ApJ, 730, 38

Wang, W.-H., Cowie, L. L., Barger, A. J., Keenan, R. C., \& Ting, H.-C. 2010, ApJS, 187, 251

Williams, R. J., Quadri, R. F., Franx, M., van Dokkum, P., \& Labbé, I. 2009, ApJ, 691, 1879

Wirth, G. D., Willmer, C. N. A., Amico, P., et al. 2004, AJ, 127, 3121

Wolf, C., Hildebrandt, H., Taylor, E. N., \& Meisenheimer, K. 2008, A\&A, 492, 933

Wolf, C., Meisenheimer, K., Kleinheinrich, M., et al. 2004, A\&A, 421, 913

Wuyts, S., Förster Schreiber, N. M., van der Wel, A., et al. 2011, ApJ, 742, 96

Wuyts, S., Labbé, I., Franx, M., et al. 2007, ApJ, 655, 51

Xue, Y. Q., Luo, B., Brandt, W. N., et al. 2011, ApJS, 195, 10 